Linköping Studies in Science and Technology

Licentiate Thesis No. 1641

\title{
Thin Film Growth Using Pulsed AND Highly IONIZED VAPOR FluXes
}

\author{
Viktor Elofsson
}

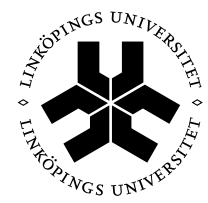

\section{Linköping University} INSTITUTE OF TECHNOLOGY

Plasma \& Coatings Physics Division

Department of Physics, Chemistry and Biology

Linköping University, Sweden

Linköping 2014 
(C) Viktor Elofsson

ISBN: 978-91-7519-426-4

ISSN: 0280-7971

Printed by LiU-Tryck, Linköping, Sweden, 2014 


\section{Abstract}

Microstructure and morphology of thin films are decisive for many of their resulting properties. To be able to tailor these properties, and thus the film functionality, a fundamental understanding of thin film growth needs to be acquired. Film growth is commonly performed using continuous vapor fluxes with low energy, but additional handles to control growth can be obtained by instead using pulsed and energetic ion fluxes. In this licentiate thesis the physical processes that determine microstructure and morphology of thin films grown using pulsed and highly ionized vapor fluxes are investigated.

The underlying physics that determines the initial film growth stages (i.e., island nucleation, island growth and island coalescence) and how they can be manipulated individually when using pulsed vapor fluxes have previously been investigated. Their combined effect on film growth is, however, paramount to tailor film properties. In the thesis, a route to generate pulsed vapor fluxes using the vapor-based technique high power impulse magnetron sputtering (HiPIMS) is established. These fluxes are then used to grow $\mathrm{Ag}$ films on $\mathrm{SiO}_{2}$ substrates. For fluxes with constant energy and deposition rate per pulse it is demonstrated that the growth evolution is solely determined by the characteristics of the vapor flux, as set by the pulsing frequency, and the average time required for coalescence to be completed.

Highly ionized vapor fluxes have previously been used to manipulate film growth when deposition is performed both normal and off-normal to the substrate. For the latter case, the physical mechanisms that determine film microstructure and morphology are, however, not fully understood. Here it is 
shown that the tilted columnar microstructure obtained during off-normal film growth is positioned closer to the substrate normal as the ionization degree of the flux increases, but only if certain nucleation characteristics are present. 


\section{PREFACE}

The work presented in this licentiate thesis is the first part of my PhD studies in the Plasma \& Coatings Physics division at Linköping University. The goal of my doctorate project is to contribute to the understanding of fundamental physical mechanisms that determine growth of thin films synthesized using time-dependent and highly ionized deposition processes. The research is financially supported by Linköping University. The results are presented in three appended papers, which are preceded by an introduction to the research field and the methods employed.

Viktor Elofsson

Linköping, January 2014 


\section{Appended PAPERS}

Paper 1

Time-domain and Energetic Bombardment Effects on the Nucleation and Coalescence of Thin Metal Films on Amorphous Substrates

D. Magnfält, V. Elofsson, G. Abadias, U. Helmersson and K. Sarakinos

J. Phys. D: Appl. Phys. 43, 215303 (2013)

Paper 2

Unravelling the Physical Mechanisms that Determine Microstructural

Evolution of Ultrathin Volmer-Weber Films

V. Elofsson, B. Lü, D. Magnfält, E. P. Münger and K. Sarakinos

Submitted for Publication

Paper 3

Tilt of the Columnar Microstructure in Off-normally Deposited Thin Films using Highly Ionized Vapor Fluxes

V. Elofsson, D. Magnfält, M. Samuelsson and K. Sarakinos

J. Appl. Phys. 113, 174906 (2013) 


\section{Author's contribution to the appended papers}

\section{Paper 1}

I was involved in planning the experiments, performed part of the film synthesis and all film characterization and analysis by spectroscopic ellipsometry. I also took part in writing the paper.

\section{Paper 2}

I was responsible for planning large part of the experiments, performed major part of the film synthesis and all film characterization and analysis by spectroscopic ellipsometry. I also wrote major part of the paper.

\section{Paper 3}

I was responsible for planning large part of the experiments and performed all depositions as well as film characterization and analysis. I also wrote major part of the paper. 


\section{ACKNOWLEDGEMENTS}

First and foremost, I would like to thank my supervisor Kostas Sarakinos for taking me on to this endeavor. Your persistent pursue to perform excellent science, with an ever so dedicated focus on physics, makes it a true privilege to working with you.

My PhD fellows Bo Lü and Daniel Magnfält for all great fun during, both relevant and (completely) irrelevant, rewarding discussions where we typically come to conclude that either we are right or the others are wrong!

Coauthors for their generous input in discussing and improving manuscripts.

Past and present members in the Plasma \& Coatings Physics Division for adding just about the right amount of craziness to a creative atmosphere.

People in Agora Materiae and other colleagues at IFM, especially those of the Thin Film Physics and Nanostructured Materials divisions, for creating a nice working environment with interesting discussions over fika.

Outside of academia, I would like to thank family and friends for support, encouragement and for bringing joy in to my life. I know that some of you are somewhat confused about what I am actually up to, but hopefully we can sort this out some day.

Last, but certainly not least, to my wonderful wife Jessica. I am thankful beyond what words can express. I love you! 


\section{CONTENTS}

$\begin{array}{ll}\text { Abstract } & \text { iii }\end{array}$

Preface v v

Appended Papers vii

$\begin{array}{ll}\text { Acknowledgements } & \text { ix }\end{array}$

1 Introduction $\quad 1$

1.1 Motivation ......................... 1

1.2 Research Goal \& Strategy . . . . . . . . . . . . . . . 3

2 Thin Film Growth 5

2.1 Initial Growth Stages . . . . . . . . . . . . . . . . . . 5

2.1.1 Surface Diffusion . . . . . . . . . . . . . . 5

2.1 .2 Nucleation . . . . . . . . . . . . . 7

2.1.3 Coalescence Processes . . . . . . . . . . . . . . . 9

2.1.3.1 Ostwald Ripening . . . . . . . . . . . . . 9

2.1.3.2 Sintering ................ 10

2.1.3.3 Cluster Migration . . . . . . . . . . . . . . 11

2.1.4 Continuous Film Formation . . . . . . . . . . . . . . . 12 
2.2 Growth Evolution . . . . . . . . . . . . . . . . . . 12

2.2.1 Thermodynamic Considerations . . . . . . . . . . . 12

2.2.2 Kinetic Considerations . . . . . . . . . . . . . . 13

2.3 Off-normal Film Growth . . . . . . . . . . . . . . . . . . 15

2.4 A Note on Stress . . . . . . . . . . . . . . . . . . . . . . 16

3 Thin Film Processes $\quad 17$

3.1 Basic Plasma Physics . . . . . . . . . . . . . . . 17

3.2 Magnetron Sputtering . . . . . . . . . . . . . . . . 18

3.3 High Power Impulse Magnetron Sputtering . . . . . . . . . . . . . 20

4 Computer Simulations $\quad 23$

5 Characterization Techniques $\quad 27$

5.1 Mass Spectrometry . . . . . . . . . . . . . . . . 27

5.2 Quartz Crystal Microbalance . . . . . . . . . . . . . . . . 28

5.3 Spectroscopic Ellipsometry . . . . . . . . . . . . . . . 28

5.3 .1 Models ....................... 31

5.3.1.1 Lorentz Oscillator . . . . . . . . . . . . 31

5.3.1.2 Drude Model . . . . . . . . . . . . . . . 33

5.3.1.3 Doremus Method . . . . . . . . . . . . . 34

5.3.1.4 Arwin-Aspnes Method . . . . . . . . . . . 35

5.4 Stress Measurements by Wafer Curvature . . . . . . . . . . . . . 37

5.5 Scanning Electron Microscopy . . . . . . . . . . . . . . . . 38

5.6 Atomic Force Microscopy . . . . . . . . . . . . . . . . . . . . 39

5.7 X-ray Reflectometry . . . . . . . . . . . . . . . . . . . 40 
6 Summary of Appended Papers

7 Future Research Possibilities

References

Papers 1-3 



\section{CHAPTER 1}

\section{INTRODUCTION}

\subsection{Motivation}

Thin films are material layers (typically $<\sim 1 \mu \mathrm{m}$ thick) that are used to cover a surface of an object - referred to as substrate - in order to enhance or alter its properties. This ability has been utilized by mankind for thousands of years [1] and is today a vital part of our everyday life. The latter manifests itself by the wide range of commercial products in which thin films are employed, such as watches, windows, transistors, hard drives, solar cells, drills and frying pans.

Many film properties are directly related to the film microstructure and morphology, which thus are decisive for their functionality. This can be understood by, e.g., considering energy saving windows where thin Ag films can be applied to reflect infrared light (heat) while letting visible light through [2]. On warm days this means that heat from the sun is blocked to maintain low temperatures inside buildings, while undesirable heat losses are prevented during cold days. This function becomes possible when Ag films are sufficiently thin to be transparent for the visible light, while at the same time they are electrically conductive to be able to reflect the infrared part of the electromagnetic spectrum. 
From the above discussion, it is evident that a fundamental understanding of thin film growth is required to be able to design and tailor film microstructure and morphology, and thus the resulting properties. Thin film growth can be initiated by condensing single atoms from the vapor phase onto a substrate. As atoms meet on the surface they nucleate and form separated atomic islands that grow in size and coalesce before forming a continuous film. These initial formation stages (i.e., island nucleation, island growth and island coalescence) set characteristic length scales of the growing films and are thus decisive for microstructural and morphological features of films, such as surface roughness, island sizes and separation, and the point at which a continuous film is formed [3, p. 495].

The most common way to grow thin films from the vapor phase is by supplying a continuous flux of atoms with low energy to the substrate surface. Additional handles to affect growth can be obtained by instead utilizing pulsed and energetic fluxes. The former affects dynamics of the growth process while the latter can activate surface and subsurface processes that are relevant for growth. Typically, energetic deposition fluxes are generated by using highly ionized fluxes [4], which allow the incoming energy to be controlled by use of electric fields. At the same time, electric and/or magnetic fields can be employed to control the trajectories of the ions. This strategy has been extensively employed over the last decades to control film growth when deposition is preformed normal to the substrate surface [4-7]. Research has also demonstrated the ability of using ionized vapor fluxes to control film microstructure and morphology when deposition is carried out at grazing incidence (off-normal growth) [8-10]. However, the fundamental physical processes that determine the growth evolution when performing off-normal deposition using ionized fluxes are not fully understood. Moreover, studies have unravelled the underlying physics that determines the effect of pulsed vapor fluxes on the initial film formation stages and how they individually can be manipulated [11-14]. Understanding their combined effect on thin film formation is, however, required to enable nanoscale design and tailoring of film properties. This knowledge is currently lacking. 
High power impulse magnetron sputtering (HiPIMS) is a vapor-based deposition technique that emerged 15 years ago from research work performed at Linköping University [15]. Up to now it has been employed almost exclusively to improve film properties owing to its capability of producing energetic ion fluxes [16]. Concurrently, it exhibits potential to generate pulsed deposition fluxes and could thus also possess the ability to be used as a tool in surface science studies in order to understand film growth processes.

\subsection{Research Goal \& Strategy}

My goal with this thesis is to contribute to the understanding of the fundamental physical processes that determine growth evolution of thin films deposited by pulsed and highly ionized vapor fluxes. This is realized by showing that HiPIMS can be utilized to generate pulsed deposition fluxes in Paper 1, using in situ plasma diagnostics and particle transport simulations. Understanding the effect of these fluxes on the initial growth stages as well as their combined effect on the film growth evolution is then acquired in Papers 1 and 2. This is accomplished by combining in situ film growth monitoring, ex situ imaging and growth simulations to study the growth all the way from nucleation to the formation of a continuous film. The physical mechanisms that determine the microstructural and morphological evolution of films grown off-normally using highly ionized vapor fluxes are then established in Paper 3, by employing ex situ imaging and particle transport simulations. 


\section{CHAPTER 2}

\section{THIN FILM GROWTH}

\subsection{Initial Growth Stages}

\subsubsection{Surface Diffusion}

As atoms from the vapor phase condense on a substrate surface they transfer most of their energy in to lattice vibrations before being adsorbed and referred to as adatoms. On the surface, adatoms experience a potential energy landscape originating from the surface atoms. Due to fluctuations in this energy landscape some sites offer more energetically stable positions than others and act as preferential adsorption sites. Adatoms are able to move between these sites if they possess sufficient energy to overcome the energy barrier for surface diffusion, $E_{D}$, that separates neighbouring sites. This process is known as surface diffusion and can be described as a two-dimensional random walk between adjacent adsorption sites. The rate at which these events occur is described by the adatom jump rate, $v$, as [17, p. 17]

$$
v=v_{0} \exp \left(-\frac{E_{D}}{k_{B} T}\right)
$$

for a certain temperature $T$. $v_{0}$ is a constant known as the attempt frequency and $k_{B}$ is the Boltzmann constant. For the typical distance covered in a single 
jump, $a$, the surface diffusion coefficient $D$ is given by the relation [17, p. 17]

$$
D=\frac{1}{4} a^{2} v=\frac{1}{4} a^{2} v_{0} \exp \left(-\frac{E_{D}}{k_{B} T}\right)
$$

where the factor $1 / 4$ accounts for the two-dimensional nature of the diffusion process. As is evident from Eq. (2.1.2) adatom diffusivity can be increased by simply raising $T$. The other main deciding factor is $E_{D}$ that is determined by the interaction between adatoms and the underlaying surface atoms. Additional contributions to $E_{D}$ can also arise from morphological features of the surface as depicted in Fig. 2.1 (a), where a step between two terraces is present. On top of terraces adatoms only experience $E_{D}$ for diffusion between adjacent sites, but a larger energy is required to descend a step since adatoms also need to break a bond. This causes an additional barrier known as the step edge barrier or Ehrlich-Schwoebel barrier, $E_{E S}$ (see Fig. 2.1 (b)). On the contrary, ascending step edge sites instead act as trapping sites since they offer a higher coordination number and thus more stable positions.

(a)

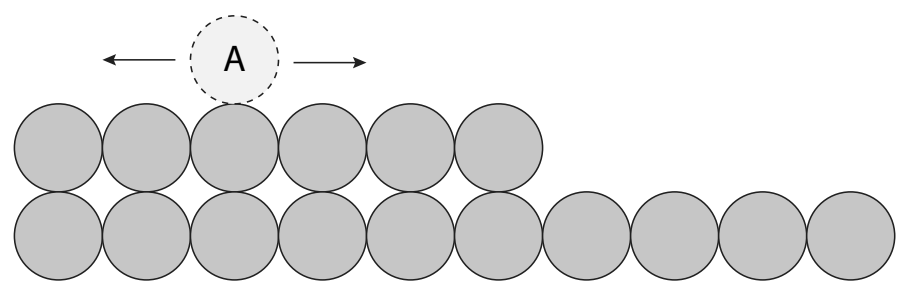

(b)

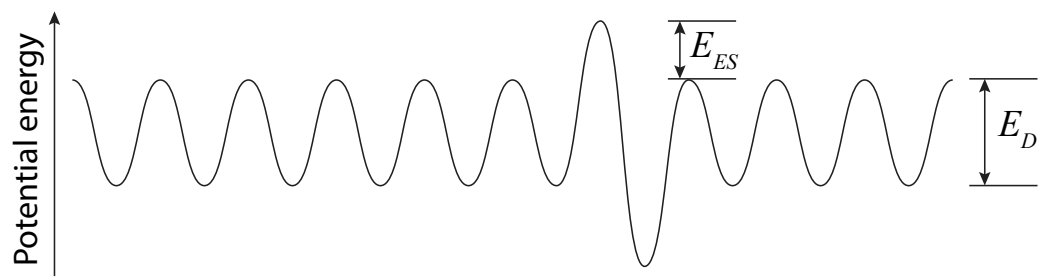

Fig. 2.1. (a) An adatom, A, on top of a terrace with possible diffusion directions indicated by arrows. (b) Energy landscape of the surface in (a) as experienced by an adatom. The diffusion barriers $E_{E S}$ (arising from the step in (a)) and $E_{D}$ are indicated in the figure. 
Even though surface diffusion of adatoms normally is considered to be a thermally activated process, it can be promoted by using low energy ion irradiation $[18,19]$. Adatom diffusion can also be increased if atoms impinge on a surface at a grazing incidence angle. In that case, they do not come to rest at the impact site, instead they exhibit a directional diffusion induced by their large momentum component along the surface [20].

\subsubsection{Nucleation}

Adatoms on an atomically flat surface can either desorb back to the vapor or diffuse until they encounter other adatoms that bind to each other and form a cluster. The cluster can, in turn, dissolve back into the two-dimensional adatom gas or form a stable nucleus. The latter occurs if the cluster size is larger than a critical value, $i^{*}$, commonly represented in number of atoms. For low growth temperatures $i^{*}$ is often equal to one [21, pp. 567-579], which means that a nucleus consisting of two or more atoms are more likely to grow than to dissociate. The nucleation process described above is typically characterized by the island density, $N$, i.e., the number of atomic islands present on the surface per unit area. In the very beginning of the growth, $N$ depends predominantly on the amount of deposited material [17, p. 28]. This is known as the transient nucleation regime and is valid until an appreciable amount of diffusing adatoms are captured by already existing islands. As this happens the steady-state nucleation regime is entered and $N$ scales as $[17$, p. 29]

$$
N \sim\left(\frac{F_{a v g}}{D}\right)^{\chi}
$$

for a continuous supply of atoms from the vapor phase. In Eq. (2.1.3) $F_{a v g}$ is the average deposition rate and $\chi$ is a scaling exponent that depends on $i^{*}$ and the dimensionality of the growth process. For three-dimensional islands grown on a two-dimensional surface $\chi$ is given by the relation [17, p. 49]

$$
\chi=\frac{i^{*}}{i^{*}+2.5^{\prime}}
$$


which yields $\chi=2 / 7 \approx 0.286$ for $i^{*}=1$. From Eq. (2.1.3) it is evident that $N$ can be increased by either increasing $F_{a v g}$ or decreasing $D$, where the latter can be accomplished by simply lowering the growth temperature (cf. Eq. (2.1.2)). It should, however, be noted that $D$ depends exponentially on $T$ (cf. Eq. (2.1.2)), which means that $F_{a v g}$ needs to be varied many orders of magnitudes to yield the same result as obtained when varying $T$.

The nucleation characteristics can also be altered by chopping a continuous vapor flux into pulses characterized by their width, $t_{o n}$, amplitude, $F_{i}$, and frequency, $f$. This yields an additional kinetic handle for nucleation depending on the interplay between the time scale of the vapor flux and the time scale of the diffusing adatoms, as characterized by the adatom lifetime, $\tau_{m}$. The latter can be approximated as [11]

$$
\tau_{m} \approx \frac{l^{2}}{D}
$$

if adatoms mainly disappear by diffusion into existing islands separated by a mean distance of $2 l$. In the case that $\tau_{m} \gg 1 / f$, adatoms are still present on the surface between successive pulses (see Fig. 2.2 (a)) and the substrate experiences a continuous deposition flux $F_{a v g}=F_{i} t_{o n} f$, which means that $N$ scales according to Eq. (2.1.3). In the other limiting case, adatom diffusion and nucleation happen almost instantly within a single pulse $\left(\tau_{m} \ll t_{\text {on }}\right.$, Fig. 2.2 (c)), which implies that the substrate sees the instantaneous flux as if it would be continuous. This means that $N$ scales as [11]

$$
N \sim\left(\frac{F_{i}}{D}\right)^{\chi}
$$

From this equation it can be understood that for fluxes characterized by $F_{i} \gg$ $F_{a v g}$ (typical for pulsed deposition) $N$ can be increased considerably. The two regimes are separated by a region where adatoms still diffuse between adjacent pulses, but disappear before the next pulse arrives $\left(t_{o n}<\tau_{m}<1 / f\right.$, Fig. 2.2 (b)). In this region $N$ is independent of $D$ and scales only with the deposition rate per pulse according to [11]

$$
N \sim\left(F_{i} t_{\text {on }}\right)^{1 / 2}
$$



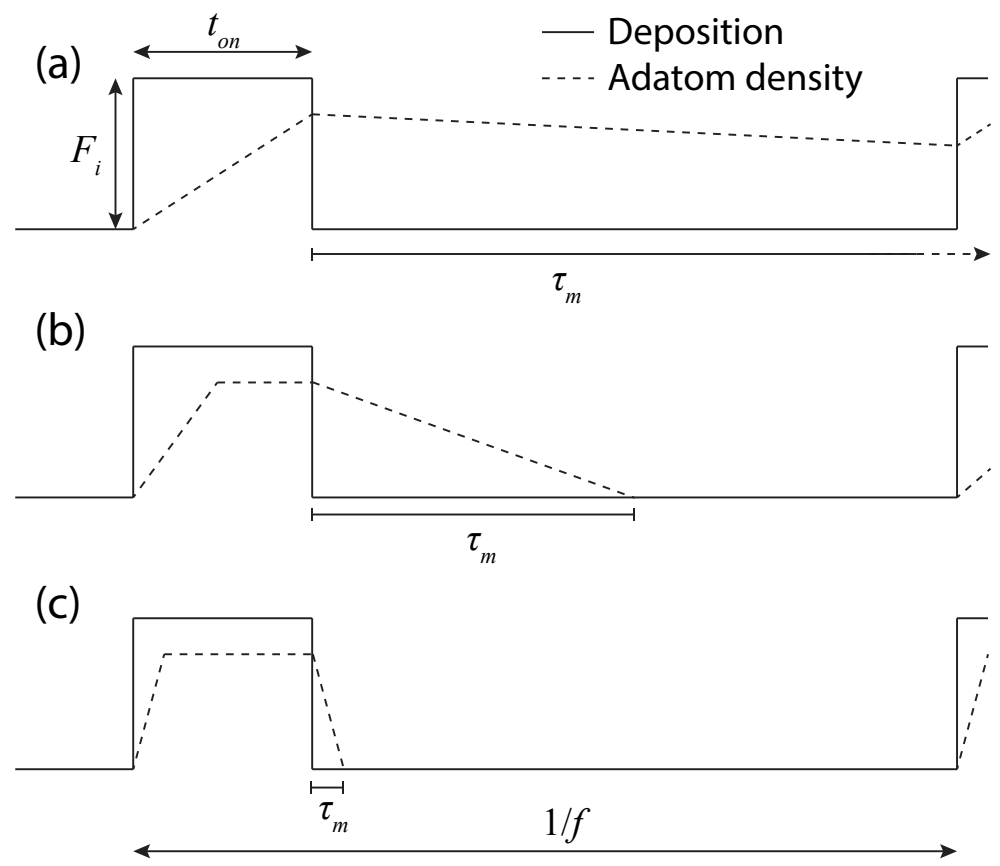

Time

Fig. 2.2. Schematics of the three different nucleation regimes in pulsed deposition. In (a) $\tau_{m} \gg 1 / f$, (b) $t_{o n}<\tau_{m}<1 / f$ and (c) $\tau_{m} \ll t_{o n}$. The solid line represents deposition and the dashed line the corresponding adatom density.

\subsubsection{Coalescence Processes}

\subsubsection{OSTWALD RIPENING}

The nucleation process leaves atomic islands with different sizes on the substrate surface, where larger islands are more energetically stable since a lower fraction of the bound atoms are present at the surface [3, p. 395]. This means that atoms are more likely to detach from smaller islands, which causes gradients in the adatom density. In turn, this results in a preferred diffusion of adatoms from small to large islands, as illustrated in Fig. 2.3. Large islands thus grow at the expense of smaller ones, decreasing the island density and increasing the average island size, in a process named Ostwald ripening. How- 


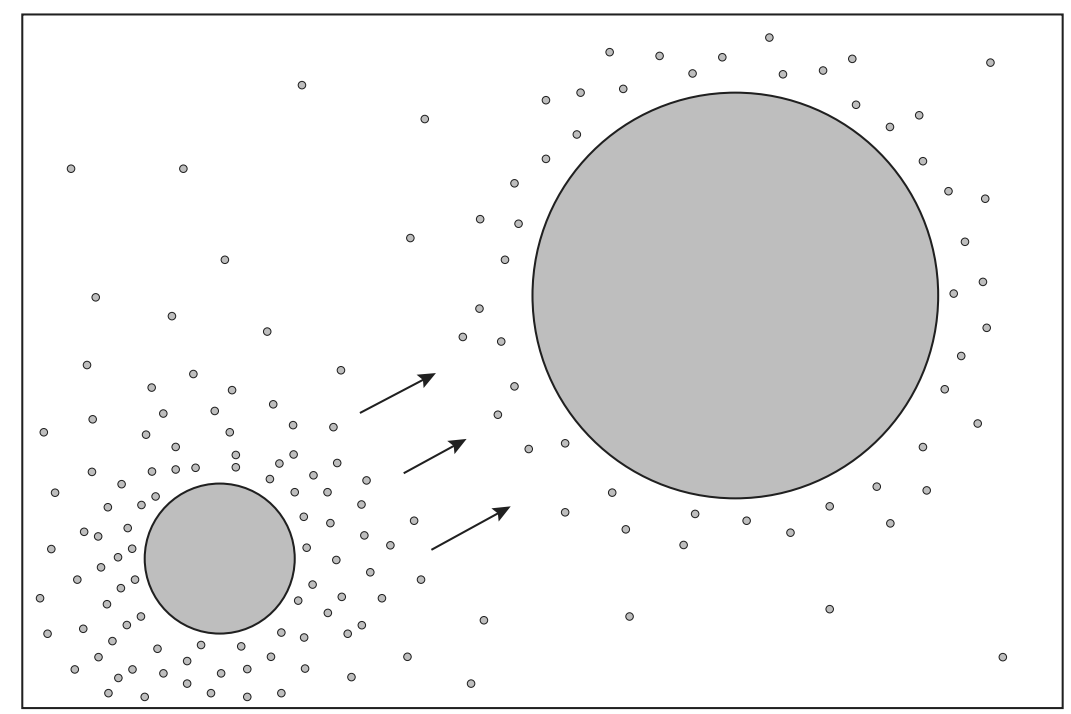

Fig. 2.3. Illustration of Ostwald ripening, where gradients in the adatom density yield a preferred adatom diffusion direction towards larger islands.

ever, this process is only of relevance if the supersaturation of adatoms is low, which commonly is not the case during thin film growth [22].

\subsubsection{SINTERING}

Adding more material to the substrate leads to growth of the atomic islands, stimulated by direct capture of atoms from the vapor phase as well as incorporation of diffusing adatoms. The distance between islands thus decreases, which, in turn, leads to island impingement at some point during growth. As this happens islands start to coalesce with each other in order to reduce the surface energy by forming a single island. This is a surface diffusion driven process caused by the large curvatures formed at the neck that connects two islands [21, p. 572, 23], as illustrated in Fig. 2.4. The time required for coalescence between two spherical or hemispherical islands with radius $R$ to be completed, $\tau_{\text {coal }}$, is given by the expression [24, 25]

$$
\tau_{\text {coal }}=\frac{R^{4}}{B}
$$



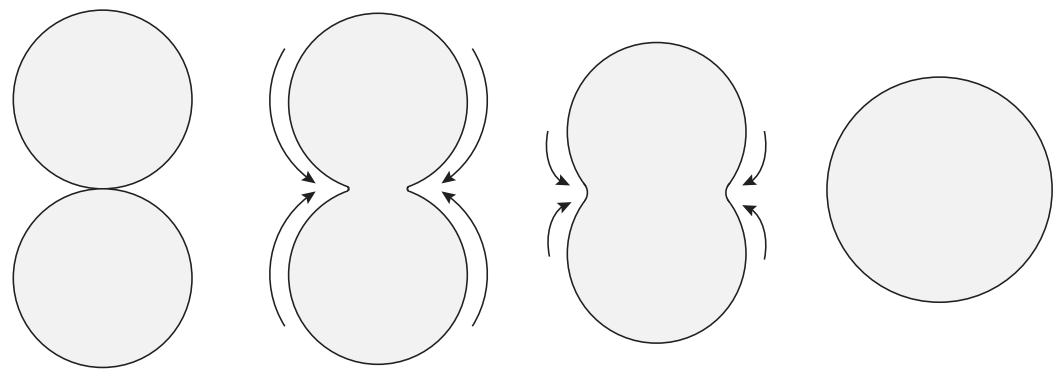

Time

Fig. 2.4. Schematic illustration of two coalescing islands.

where $B$ is a material and temperature dependent coalescence parameter given by the relation $[24,25]$

$$
B=\frac{D_{s} \gamma \Omega^{2} S_{0}}{k_{B} T} .
$$

In Eq. (2.1.9) $D_{S}$ is the diffusion coefficient for atom diffusion on the island, $\gamma$ the surface energy, $\Omega$ the atomic volume and $S_{0}$ the number of diffusing atoms per unit surface area. If, however, grain boundaries are formed between two impinging islands, e.g., if they exhibit different crystallographic orientations, the grain boundary needs to diffuse out in order for coalescence to be completed, impeding coalescence [25]. Moreover, given that coalescence is completed a denuded region is formed around the new single island that enables additional nucleation events to take place in the exposed area. This is referred to as secondary nucleation.

\subsubsection{Cluster Migration}

Coalescence between islands can also take place even when deposition does not lead to island growth and impingement, as described in the previous section (Sec. 2.1.3.2). This can happen if small islands exhibit sufficient mobility to be able to diffuse on the surface. In that case, diffusing islands can coalesce with other islands that are encountered as they migrate on the surface. It should 
be noted that island diffusion typically is considered to only be able to occur for smaller sized islands consisting of less than tens of atoms, with an island diffusivity that normally decreases with increasing island size, even though diffusion of larger islands with hundreds of atoms has been observed [26].

\subsubsection{Continuous Film Formation}

At some point during growth, as islands grow larger, island coalescence is not completed before a third island impinges on the coalescing cluster since $\tau_{\text {coal }}$, in accordance to Eq. (2.1.8), increases with island size. This forms elongated island structures separated by voids on the substrate surface. Further deposition causes more and more of these structures to join together forming networks of connected islands. The latter enables free electrons in metallic films to travel longer distances, yielding electrical conductivity. A continues film is then formed as the voids are filled in.

\subsection{Growth Evolution}

The route that leads to formation of continuous films and the growth thereafter can take different paths depending on the interaction between substrate and film as well as the conditions at which the films are grown. This results in different characteristic growth modes of the films that cause different microstructures and morphologies. These growth modes are discussed and explained in the following section.

\subsubsection{Thermodynamic Considerations}

The effect of thermodynamics on the microstructural evolution during epitaxial growth ${ }^{1}$ is dictated by the relationship between the surface energy of the

\footnotetext{
${ }^{1}$ The following growth modes were developed for epitaxial systems, but an extension to also include polycrystalline film growth is conceivable.
} 
substrate $\left(\gamma_{s}\right)$, the film $\left(\gamma_{f}\right)$ and the film-substrate interface $\left(\gamma_{i}\right)$. Typical for epitaxial growth is that $\gamma_{s} \geq \gamma_{f}+\gamma_{i}$, which means that the film completely wets the substrate in order to minimize the surface energies and hence grows in a layer-by-layer fashion (also known as the Frank van der Merwe growth mode, Fig. 2.5 (a)). For the case of homoepitaxy equality holds in the above relation since $\gamma_{i}=0$ [27, p. 18]. On the contrary, if $\gamma_{s}<\gamma_{f}+\gamma_{i}$ the surface energy is instead minimized by bunching up and growing islands of the deposit (referred to as Volmer-Weber growth mode, Fig. 2.5 (b)). A third growth mode also exists where the film starts to grow in a layer-by-layer fashion, but after a critical thickness strain relaxes by formation of three dimensional islands. This is known as the Stranski-Krastanov growth mode (Fig. 2.5 (c)).

(a)

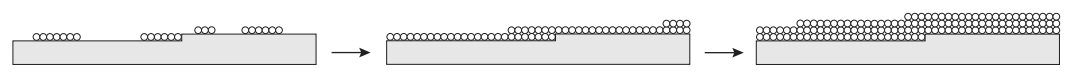

(b)

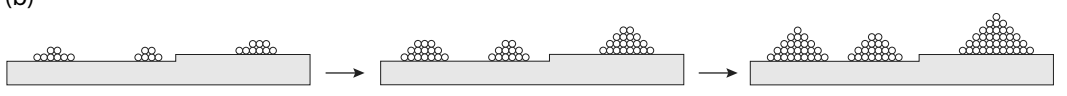

(c)

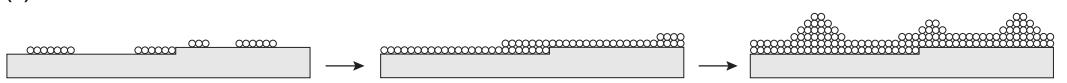

Fig. 2.5. Illustration of different growth modes depending on the surface energies. (a) Frank van der Merwe (layer-by-layer), (b) Volmer-Weber (island formation) and (c) Stranski-Krastanov (layer-plus-island) growth mode.

\subsubsection{Kinetic Considerations}

Thin films deposited from the vapor phase are often grown far from conditions of thermodynamic equilibrium described in the previous section (Sec. 2.2.1), which means that kinetic processes also affect the growth evolution. This can easily be understood by considering the importance of the diffusion barriers $E_{D}$ and $E_{E S}$ during homoepitaxial film growth that, from the viewpoint of thermodynamics, exhibit potential to grow as layer-by-layer. In the case that adatom 
diffusion is high enough to overcome both $E_{D}$ and $E_{E S}$ and that the diffusion length is much longer than the terrace width, adatoms are able to descend steps and are thus incorporated at step edges. This leads to a step flow growth where no nucleation events takes place (Fig. 2.6 (a)). For diffusivities high enough to still overcome both $E_{D}$ and $E_{E S}$, but with a considerably shorter diffusion length, two-dimensional islands nucleate on top of terraces. Since adatoms captured on top of islands descend island edges, a complete layer is formed before any new nucleation events takes place, which results in a layer-by-layer growth (Fig. 2.6 (b)). On the other hand, if $E_{E S}$ hinders interlayer transport of adatoms three-dimensional mounds are formed on the terraces (Fig. 2.6 (c)), leading to kinetic roughening. Moreover, in the case of no intralayer diffusion (adatoms do not overcome $E_{D}$ ), diffusion is in practice turned off, and adatoms come to rest where they are deposited. This is known as self-affine growth and leads to an open structure with a large surface roughness (Fig. $2.6(\mathrm{~d})$ ).

(a)

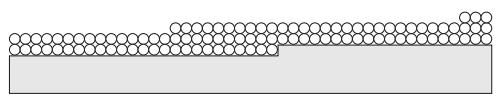

(c)

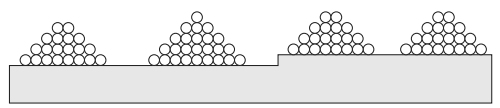

(b)

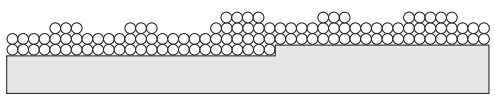

(d)

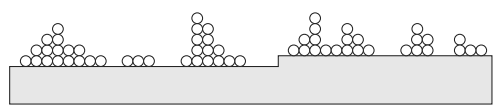

Fig. 2.6. Illustration of different growth modes obtained due to differences in the adatom diffusivity. (a) Step flow growth, (b) layer-by-layer growth, (c) kinetic roughening and (d) self-affine growth.

In this thesis work the growth of metal films on insulators $\left(\mathrm{SiO}_{2}\right)$ are investigated. This type of system typically grows as three-dimensional islands (VolmerWeber type growth) due to weak interactions between film and substrate as well as low substrate surface energies as compared to that of the films [21, p. 569]. In addition, kinetic effects can promote three-dimensional growth as discussed above. E.g., for the case of $\mathrm{Ag}$ diffusion on $\mathrm{Ag}(111)$, which is the low 
energy plane for $\mathrm{Ag}$ [28], $E_{D} \approx 0.10 \mathrm{eV}$ [29] and $E_{E S} \approx 0.13 \mathrm{eV}$ [30]. This means that a bit more than twice the energy is needed for an adatom to descend a step as compared to for diffusion between adsorption sites. Adatoms can thus be trapped on top of islands and hence cause islands to grow threedimensionally. Other kinetic conditions do also affect the growth and can for the case of low adatom diffusivities (or equivalently high deposition rates) lead to large island densities, in accordance to Eq. (2.1.3), with an apparent twodimensional growth as result. The three-dimensional structure is highly relevant during growth of thin films and supported nanoparticles, and find technological applications in, e.g., optics [31], catalytic systems [32] and magnetic devices [33].

\subsection{Off-normal Film Growth}

After the formation of a continuous film in the case of three-dimensional growth (Volmer-Weber type growth), each grain grows in the direction of the substrate normal as more atoms are supplied from the vapor. This leads to a columnar microstructure where columns are separated by grain boundaries, as illustrated in Fig. 2.7 (a). If the flux instead arrives at an angle with respect to the substrate normal the initially formed islands shadow the area behind them, hindering further deposition to take place in this region. This yields growth of columns separated by voids that are tilted towards the vapor source, as depicted in Fig. 2.7 (b). The resulting tilt angle of the columns depends on the incidence angle of the vapor flux, but it can also be altered by changing film composition [34], spatial distribution of the vapor flux [35-37] or the kinetic growth conditions [38-42]. Yet another way to affect the column tilt is to use highly ionized vapor fluxes, as previously has been shown in e.g., Ref. [43]. This approach is systematically investigated in Paper 3, where it is demonstrated that a higher ionization degree leads to columns positioned closer to the substrate normal, but only if certain nucleation characteristics are present. 
(a)
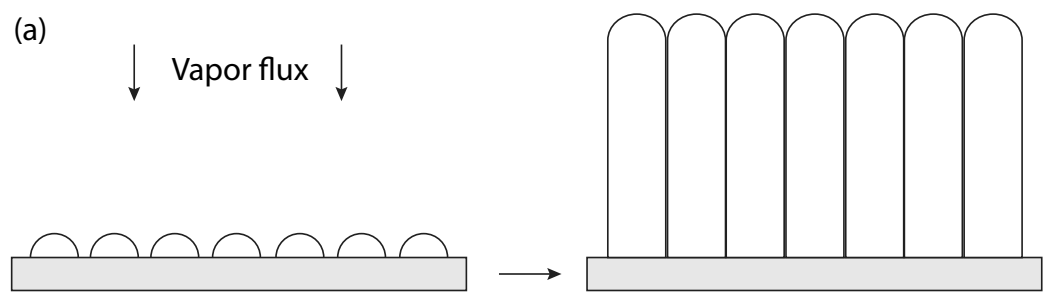

(b)

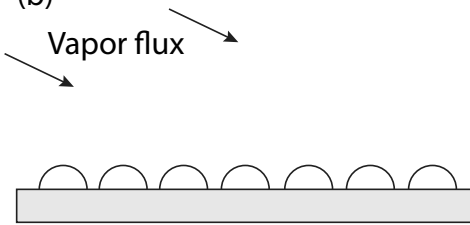

Nucleation

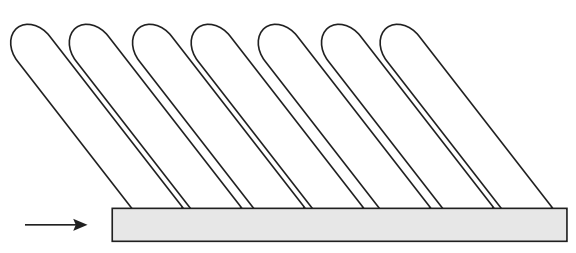

Growth

Fig. 2.7. (a) Normal incidence of the vapor flux that yields growth of a columnar microstructure in the direction of the substrate normal. (b) Growth of tilted columns due to a grazing incidence angle of the vapor flux.

\subsection{A Note on Stress}

Stresses commonly develop in thin films as they grow. They can be generated from, e.g., lattice miss match between substrate and film, point defects in the crystal lattice, and attraction or repulsion of adjacent grains over grain boundaries. Stresses also arise during the growth evolution of Volmer-Weber thin films where a compressive-tensile-compressive behaviour typically is observed for materials exhibiting high diffusivity (like $\mathrm{Ag}$ on $\mathrm{SiO}_{2}$ as investigated in Papers 1 and 2) [44]. The initial compressive stress corresponds to island nucleation and growth, while the subsequent change into tensile stress is caused by attractive forces between coalescing islands [44]. A continuous film is then formed at the point where the stress changes and once again turns into a compressive state [45], which has been suggested to be caused by grain boundary densification [46-48]. This point was employed in Paper 1 as a way to denote the formation of a continuous Ag film. 


\section{CHAPTER 3}

\section{Thin Film Processes}

The atomic vapor used to supply growing thin films with material can be generated by different means. In physical vapor deposition (PVD) the vapor is obtained by vaporizing a solid source material by physical means in a vacuum environment. The most straightforward way to do this is to supply thermal energy as heat until the source material starts to evaporate. Another way is to employ momentum in terms of energetic ions to bombard and eject atoms from a solid source - referred to as target - into the vapor phase. This process is known as sputtering and is usually associated with the use of a plasma.

\subsection{Basic Plasma Physics}

A plasma can be described as a quasi neutral, ionized gas that consists of neutral atoms, ions and electrons [49, p. 3]. As it is confined within a vacuum chamber the quasi neutrality, i.e., that the electron and ion densities are the same when averaged over a large volume, is no longer valid where the plasma gets in contact with a conductive surface. This is due to a higher electron velocity that causes more frequent losses of electrons to the surface, resulting in a positive net charge density in a region close to the surface that is named sheath. Beyond the sheath the bulk plasma is entered. Due to the sheath, the plasma 


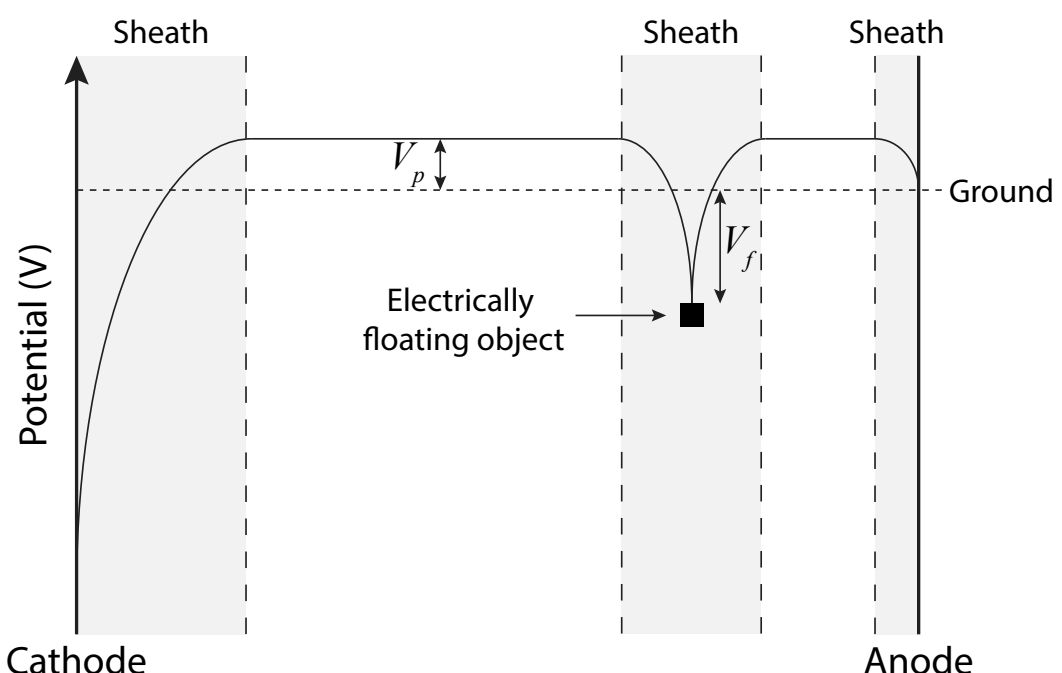

Fig. 3.1. Typical potential of a plasma used in a sputtering process. An external potential is applied to the cathode and the anode is connected to ground.

exhibits a positive potential (plasma potential, $V_{p}$ ) with respect to the conductive surface, as is illustrated in Fig. 3.1. The value of $V_{p}$ is typically around $1-5$ $\mathrm{V}$ in plasma based sputtering processes [50-53]. A similar situation arises if a conducting object, shielded from other potentials, is immersed into a plasma. In that case, the faster electrons start to build up a negative charge of the object. This means that ions are attracted to and electrons are repelled away from the object. As the net current is zero no further charging takes place, yielding a negative potential known as floating potential, $V_{f}$ (see Fig. 3.1). The latter typically takes values between -10 and $-20 \mathrm{~V}[50,54-56]$.

\subsection{Magnetron Sputtering}

In order to deposit thin films by plasma based processes an inert gas, typically Ar, is let into a vacuum chamber after which a negative potential is applied to the target (cathode). This causes free electrons ${ }^{1}$ close to the cathode to be

\footnotetext{
${ }^{1}$ Some free electrons are always present in a gas, e.g., caused by background radiation.
} 
accelerated towards the anode. On their way, they can undergo inelastic collisions with neutral Ar atoms causing impact ionization ${ }^{2}$ that in addition generate more free electrons. The latter gives rise to an electron avalanche that is accelerated towards the anode, causing further ionization events. This leads to breakdown of the gas and creation of a plasma. At the same time, the generated ions are attracted and accelerated towards the cathode. As they collide with the surface of the target, energy and momentum are transferred to atoms in the surface region through a collision cascade that can yield ejection of atoms from the target, i.e., sputtering. This process can also create secondary electrons that are important in order to sustain the plasma.

The efficiency of the sputtering process can be further increased by applying magnets behind the target as illustrated in Fig. 3.2. By doing so, charged particles experience the Lorentz force, $\vec{F}_{L}$, given as $[49$, p. 21]

$$
\overrightarrow{F_{L}}=q(\vec{E}+\vec{v} \times \vec{B})
$$

where $q$ is the particle charge, $\vec{E}$ the electric field, $\vec{v}$ the velocity of the particle and $\vec{B}$ the magnetic field. The addition of the $\vec{B}$ field thus traps electrons close to the cathode. Ions, on the other hand, are only weakly affected by $\vec{F}_{L}$ due to their larger mass [21, p. 46], but are nonetheless confined in the vicinity of the target region in order to maintain the quasi neutrality of the plasma. The plasma based deposition technique direct current magnetron sputtering (DCMS) utilizes this setup together with a constant power at the cathode to generate a continuous supply of atoms to the substrate. The vapor flux created by DCMS consists mainly of neutral atoms (only a few percent of the flux is typically ionized [57]) due to low plasma densities $\left(<\sim 10^{16} \mathrm{~m}^{-3}[16,58]\right)$ that yield low probabilities for ionization of sputtered atoms.

\footnotetext{
${ }^{2}$ Other important ionization events in the framework of this thesis are charge exchange ionization and penning ionization, where an excited atom transfers energy to a neutral atom that leads to ionization.
} 


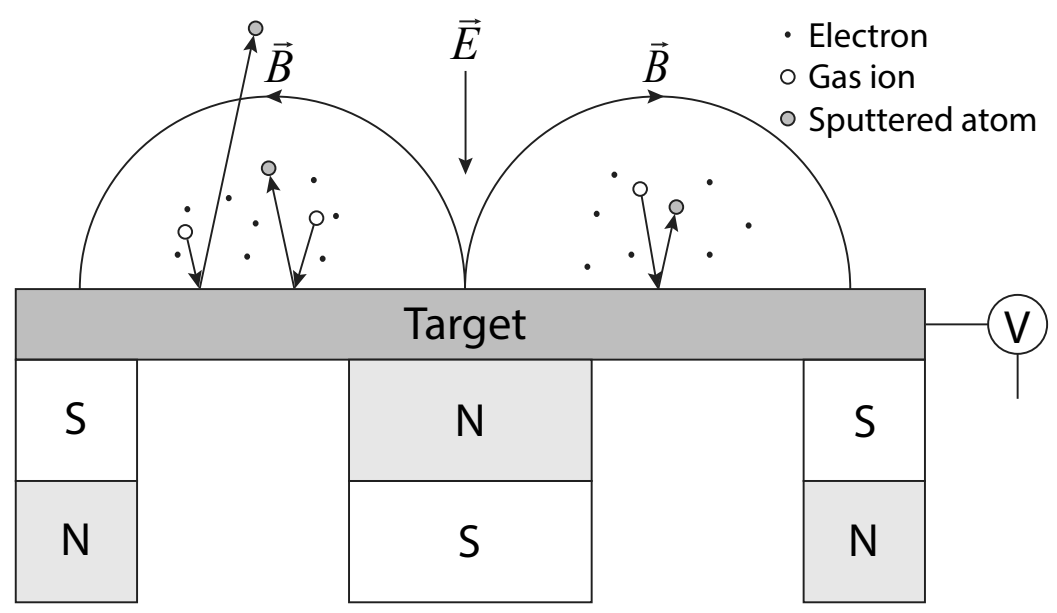

Fig. 3.2. Schematic cross-sectional view of a magnetron sputtering source.

\subsection{High Power Impulse Magnetron Sputtering}

Higher ionization degrees of the sputtered species are desired since energies and trajectories of ions can be controlled to a much higher degree than the corresponding quantities for neutrals. One way to manipulate ions is to simply apply a negative potential to the substrate causing a potential difference between bulk plasma and substrate that accelerates ions towards the growing film ${ }^{3}$. The latter can change the angular distribution of the flux and trigger surface processes that makes it possible to grow dense and smooth films, even on complex-shaped substrates. The need for higher ionization degrees during magnetron sputtering lead to the emergence of the deposition technique high power impulse magnetron sputtering (HiPIMS) [15, 16, 58-62]. In HiPIMS the power is delivered to the cathode in well-defined pulses with low duty cycles $(<10 \%)$ and frequencies lower than $\sim 10 \mathrm{kHz}$, which allows heat to be dissipated between pulses to avoid target melting. This makes it possible to obtain high plasma densities $\left(\sim 10^{19} \mathrm{~m}^{-3}\right.$ [63]) with ionization degrees of the sputtered material close to $100 \%$ [64] depending on the target material and the process

\footnotetext{
${ }^{3}$ The same is also valid for electrically floating or even grounded substrates due to the positive potential of the plasma, $V_{p}$.
} 
conditions. The ionzed species do also normally exhibit higher energies ( $\sim$ tens of $\mathrm{eV}$ ) than neutrals ( $\sim \mathrm{a}$ few $\mathrm{eV}$ ). This has been suggested to be due to temporal variations of the space charge within the plasma, which, in turn, leads to local electric fields that cause acceleration of charged species $[65,66]$.

The high ionization degree is also one of the main drawbacks of HiPIMS as compared to conventional DCMS since part of the ionized species might not possess sufficient energy to overcome the cathode sheath potential and are instead attracted back to the target. The latter causes sputtering that due to a normally lower self-sputter yield as compared to the sputter yield of the inert process gas [16] results in a loss of deposition rate. Moreover, in Paper 1 we showed that the use of HiPIMS leads to a pulsed deposition flux with a frequency corresponding to that applied to the cathode. The width of each vapor pulse is, however, slightly longer than the active part of the pulse due to scattering of the sputtered species in the gas phase $[67,68]$. 


\section{CHAPTER 4}

\section{COMPUTER Simulations}

Computer simulations are today utilized in a wide range of areas, such as finance, military and science, to predict or understand outcomes or strategies. In the field of materials science, simulations can be employed to bridge the gap between atomistic processes and macroscopic properties in order to explain or anticipate how they correlate with each other. For the sake of this thesis, where the area of interest is thin film growth, it is desired to be able to perform simulations of the growth process in real time. This implies that two different main methods can be employed for the simulations; molecular dynamics (MD) and kinetic Monte Carlo (KMC). The former is based on Newton's equations of motion that are solved numerically for each time step. In order to be able to follow the motion of individual atoms the time step thus need to be sufficiently short (typically $\sim 10^{-15} \mathrm{~s}[69, \mathrm{p} .2]$ ) to resolve atomic vibrations. This limits MD simulations to short total simulation times (typically up to $\sim 10^{-6} \mathrm{~s}[69, \mathrm{p} .2]$ ), which makes them unfeasible for the time scales under consideration in this thesis work. KMC simulations circumvent this problem by, instead of the deterministic approach used in MD, employing a stochastic approach where probabilities are assigned to different surface processes. For adatom diffusion this means that it is only the transition, in itself, between two sites that is considered, instead of keeping full track of an atom's motion between the sites. This enables a dramatic increase of the total simulation time. 
During each time step in the KMC simulations a possible event is chosen randomly and carried out. Any implications arising from the executed event is then analyzed before the time is increased one step and a new random event is chosen. For the film growth simulations in Paper 2 this means that one of two main events can occur during each time step; addition of a new adatom through deposition or movement of an already existing adatom by diffusion. The real time is thus determined by the arrival rate of new adatoms and the adatom diffusivity (set by Eq. (2.1.2)). If a diffusion event is chosen the selected adatom moves randomly to any of the four nearest-neighbour positions on a squared substrate lattice ${ }^{1}$ with equal probability in all directions, i.e., $E_{D}$ is the same in all directions. At its new position the adatom can form a new nucleus if an adjacent adatom is present (assuming $i^{*}=1$ ), get incorporated into an existing island or simply stay at rest. Similarly, if an atom is deposited it can get incorporated in an existing island by direct capture, create a new nucleus or become a free adatom. For impinging islands the coalescence time is calculated in accordance to Eq. (2.1.8) and if this time is reached a new island is formed from the coalescing islands. This procedure is then repeated until the end of the simulation.

Throughout the simulations used in Paper 2, the number of islands on the substrate surface and their size (number of atoms) as well as the number of nucleation, island impingement and completed coalescence events are accumulated to characterize the growth process. The number of islands is then used to extract a ratio between the total number of islands and the total number of clusters $^{2}$. As this ratio equals two each cluster consists, on average, of two islands, which is equivalent to elongated island structures. This point during growth is thus referred to as elongation transition [70]. The thickness at which this happens, the elongation transition thickness, is used as a comparative measure between different simulations to study scaling behaviours for different growth

\footnotetext{
${ }^{1}$ Since a finite substrate is used periodic boundary conditions are employed.

${ }^{2}$ A cluster consists of interconnected islands, where the smallest sized cluster is defined as a single island.
} 
processes. In addition, the number of events are used to calculate rates of the corresponding processes in order to determine the role of island nucleation, island growth and island coalescence on the growth evolution. 


\section{CHAPTER 5}

\section{ChaRACTERIZATION TECHNIQUeS}

\subsection{Mass Spectrometry}

Mass spectrometry is an analysis technique capable of measuring atomic masses and energies. It is utilized in thin film processes to provide quantitative information of plasma chemistry and energies.

An extraction probe with a small orifice ( tens to hundreds of $\mu \mathrm{m}$ in diameter) is immersed into the plasma through which gas atoms and ions may enter the mass spectrometer. The incoming species are then filtered depending on their energy and mass-to-charge ratio using electric and magnetic fields before being detected. This implies that neutral species first need to be ionized in order to be studied. Commonly, this is accomplished by employing a heated filament that eject electrons, causing ionization of the neutrals upon impact.

In Paper 1, a Bessel box is employed as energy filter. It uses electrodes with applied potentials to only allow ions with a specific energy to pass through. As mass filter, a quadrupole is utilized. It employs four parallel metal rods, onto which a constant as well as a time-varying potential is applied. Depending on the potentials only ions with a certain mass-to-charge ratio are able to travel through the filter without colliding with the rods and finally reach the detector. 
Mass spectrometry is utilized in Paper 1 to study the pulsed nature of the ionized deposition flux generated in the HiPIMS process (see Sec. 3.3) as well as the corresponding ion energies.

\subsection{Quartz Crystal Microbalance}

A quartz crystal microbalance (QCM) is an instrument that is capable of detecting a small addition or removal of mass. Thanks to its ease of use it is widely employed to monitor deposition rates during thin film growth.

The QCM is based on the piezoelectric effect where an applied mechanical strain generates an electric field across a piezoelectric material. In the same way, applying an electric field to the same material causes a mechanical strain that restores as the field is removed, i.e., an elastic deformation. By applying an alternating potential, the crystal starts to oscillate and a standing wave can be generated. The corresponding resonant frequency of the wave is highly sensitive to changes in the thickness of the crystal, which means that if an additional layer is deposited on top of the crystal the resonant frequency shifts. Assuming a uniform and rigid layer the shift of the resonant frequency can be directly correlated to the mass change per unit area through the Sauerbrey equation [71]. With knowledge about the film density the deposition rate can be extracted. The latter is done in Paper 3 for one deposition set to get the same deposition rate for all deposition conditions.

\subsection{Spectroscopic Ellipsometry}

Spectroscopic ellipsometry is a surface sensitive optical characterization technique that measures changes in the polarization state of light that is reflected from a material. It is typically used to give information about optical properties and film thicknesses, but can also be utilized to determine other material properties, such as surface and interface roughnesses, electrical resistivity and 


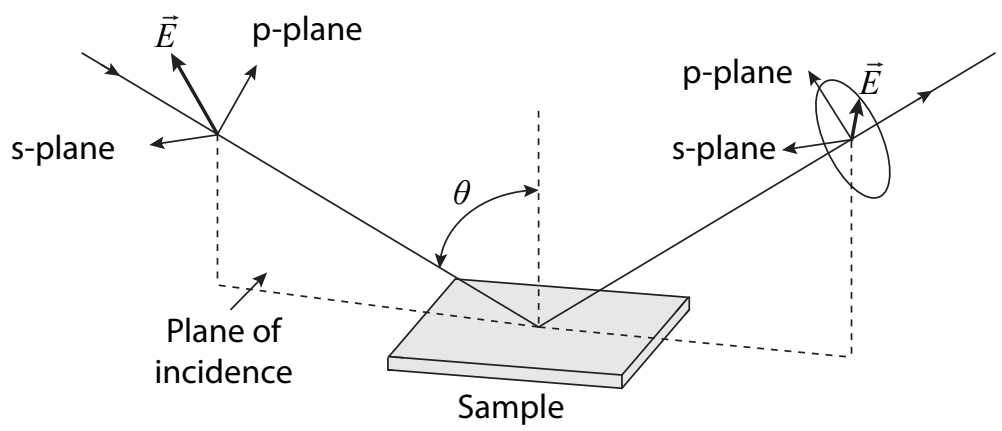

Fig. 5.1. Setup for spectroscopic ellipsometry measurements where linearly polarized light illuminates a sample and is reflected back as elliptically polarized.

chemical composition. A main attribute of this technique, for the sake of the work conducted in this thesis, is the capability of using it in situ to acquire real time measurements during thin film growth.

In spectroscopic ellipsometry, a sample is illuminated by electromagnetic waves (light) with a known polarization ${ }^{1}$ at multiple energies, $\omega$, in the visible or close to the visible spectrum (see Fig. 5.1). The interaction between the electromagnetic waves and the electrons in the sample determines the optical response of the material, which is characterized by the material's complex dielectric function, $\tilde{\epsilon}=\tilde{\epsilon}(\omega)$. $\tilde{\epsilon}$ is normally separated into its real and imaginary parts and is then expressed as

$$
\tilde{\epsilon}=\epsilon_{1}+i \epsilon_{2}
$$

$\epsilon_{1}$ and $\epsilon_{2}$ are correlated with each other through the Kramers-Kronig relations ${ }^{2}$ and related to the complex index of refraction, $\tilde{n}=n+i \kappa$, as [72, p. 430]

$$
\begin{gathered}
\epsilon_{1}=n^{2}-\kappa^{2} \\
\epsilon_{2}=2 n \kappa .
\end{gathered}
$$

\footnotetext{
${ }^{1}$ Linearly polarized light is typically used, but any kind of polarization can be employed as long as it is known.

${ }^{2}$ The Kramers-Kronig relations are mathematical relationships that correlate the real and imaginary part of a complex function. I.e., if one part is known the other can be calculated.
} 
Here, $n$ is the refractive index, signifying the phase speed of the light in the material, and $\kappa$ is the extinction coefficient that describes light absorption. For a bulk material, $\tilde{n}$ is related to the Fresnel reflection coefficients, $r_{p}$ and $r_{s}$, where $p$ and $s$ refer to the planes that are parallel and perpendicular to the plane of incidence, respectively (see Fig. 5.1). They are the normalized electric field components, with respect to the incident electric field, in the $p$ and $s$ planes and thus a measure on the change in polarization for each direction. The latter typically happens differently in the $p$ and $s$ directions causing the reflected light to be elliptically polarized.

The ratio of $r_{p}$ and $r_{s}$ is called the complex reflectance ratio, $\rho$, which is the quantity of interest in spectroscopic ellipsometry measurements. It is related to the experimentally determined ellipsometric angles $\Psi$ and $\Delta$ by the relationship [73]

$$
\rho=\frac{r_{p}}{r_{s}}=\tan \Psi e^{i \Delta}
$$

Obtaining $\rho$ then enables one to calculate $\tilde{\epsilon}$ as [74, p. 280]

$$
\tilde{\epsilon}(\omega)=\tilde{n}_{0}^{2} \sin ^{2} \theta\left(1+\left(\frac{1-\rho(\omega)}{1+\rho(\omega)}\right)^{2} \tan ^{2} \theta\right)
$$

where $\tilde{n}_{0}$ is the complex index of refraction of the sample's ambient (for vacuum $\tilde{n}_{0}=1$ ) and $\theta$ the incidence angle of the light with respect to the sample surface normal. The above equations are, however, valid for bulk materials where transmitted light is totally absorbed within the material. This is not the case for thin films grown on bulk substrates where part of the light is reflected back at the film-substrate interface. The latter causes light to once again travel through the film before reaching its surface where both reflection and transmission occur. Due to these multiple reflections the optical response of the three phase system (ambient-film-substrate, denoted as medium 0-1-2) is instead determined by the total Fresnel reflection coefficients, $R_{p}$ and $R_{s}$, given as [74, $\mathrm{p}$. 282]

$$
R_{p}=\frac{r_{01 p}+r_{12 p} e^{i 2 \beta}}{1+r_{01 p} r_{12 p} e^{i 2 \beta}}
$$




$$
R_{s}=\frac{r_{01 s}+r_{12 s} e^{i 2 \beta}}{1+r_{01 s} r_{12 s} e^{i 2 \beta}} .
$$

Here, $r_{01 j}$ and $r_{12 j}$ are the Fresnel reflection coefficients for the ambient-film (medium 0 to 1 ) and the film-substrate (medium 1 to 2 ) interface, respectively, and $\beta$ the phase angle that is defined as [74, p. 282]

$$
\beta=\frac{2 \pi d}{\lambda} \sqrt{\tilde{n}_{1}^{2}-n_{0}^{2} \sin ^{2} \theta}
$$

In the above equation, $d$ is the film thickness and $\lambda$ the wavelength of the light in vacuum. The expression of $\rho$ thus changes in to

$$
\rho=\frac{R_{p}}{R_{s}}
$$

which infer that $\rho$ now carries information about the entire three-phase system, i.e., the corresponding complex dielectric functions as well as $d$. This means that a combined complex dielectric function for the whole system, known as the pseudo-dielectric function, is obtained when using Eq. (5.3.4).

\subsubsection{Models}

Spectroscopic ellipsometry is an indirect characterization technique in the sense that physical properties are determined by modelling the sample under investigation. In this section the models used to represent the optical response of the film during this thesis work are presented.

\subsubsection{LORENTZ OSCILLATOR}

If an external electric field, $E(t)=E_{0} e^{-i \omega t}$, is applied to a material, a force is exerted on the bound electrons that can induce displacement of the electron clouds. The displacement, $x$, of a single electron with charge $e$ and mass $m_{e}$ can classically be described by its equation of motion when it is considered to be attached to a fixed ion (due to its heavier mass) through a damped spring, 
according to $[73,75$, p. 43$]$

$$
m_{e} \frac{d^{2} x}{d t^{2}}=-m_{e} \Gamma \frac{d x}{d t}-m_{e} \omega_{0}^{2} x-e E(t)
$$

In the equation, $\Gamma$ is a damping constant that accounts for electron scattering and $\omega_{0}$ is the resonance frequency of the electron oscillations. In turn, the electron displacement causes the formation of a dipole and thus determines the polarization of the atoms. Therefore, a material's polarization can be obtained by solving the equation of motion for a harmonic oscillator, which also gives an expression of $\tilde{\epsilon}(\omega)$ as $[73,75$, p. 45]

$$
\tilde{\epsilon}(\omega)=\epsilon_{\infty}+\frac{e^{2} N_{e}}{\epsilon_{0} m_{e}} \frac{1}{\omega_{0}^{2}-\omega^{2}-i \Gamma \omega} .
$$

Here, $\epsilon_{\infty}$ is a constant that accounts for contributions to $\tilde{\epsilon}(\omega)$ from interband transitions that commonly occur at higher energies and for the case of Ag starts at $3.8 \mathrm{eV}$ [76]. The other unknown parameters in Eq. (5.3.9) are the number of electrons per unit volume $N_{e}$ and the permittivity of free space $\epsilon_{0}$. This is known as the Lorentz oscillator.

The applicability of using a Lorentz oscillator to describe the optical response of thin films can be understood by considering a non-continuous film consisting of separated atomic islands (with metallic behaviour) present on a substrate surface. In that case, an island can be considered to consist of fixed positively charged ions with freely moving conduction electrons, confined within each island (as depicted in Fig. 5.2 (a)). As an electric field is applied across an island the conduction electrons are accumulated on one side of the island, creating a dipole. This gives rise to an electric field inside the island that is opposite to the one applied (see Fig. 5.2 (b)), which, in turn, generates a restoring force on the electrons that aims at bringing them back to their equilibrium positions. If the external electric field is removed at some point electrons start to oscillate collectively at a resonant frequency. The oscillations should ideally continue for an infinite amount of time, but in reality they cease due to damping from 
- Electron

- Ion

(a)

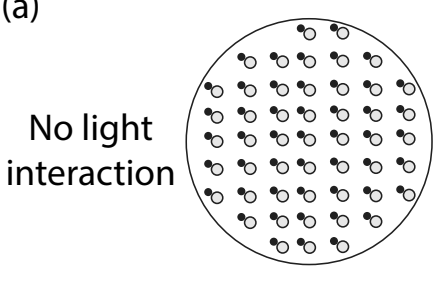

(b)

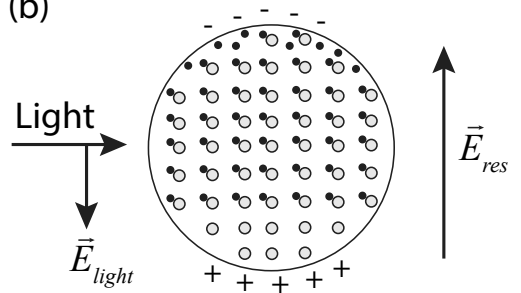

Fig. 5.2. Schematics of a metallic island (a) without and (b) with light interaction. In (b) electrons move opposite to the applied electric field, $\vec{E}_{\text {light }}$, which, in turn, creates a restoring field inside the island, $\vec{E}_{\text {res }}$.

the ions and the island surface. These collective electron oscillations, known as localized surface plasmon resonances [31], are thus similar to a harmonic oscillator with damping and, hence, well described by the Lorentz oscillator. In Paper 2 one Lorentz oscillator was used to describe the optical response of noncontinuous Ag films in order to extract the resonance energy, $\omega_{0}$, signifying the absorption maximum.

As islands get closer to each other they start to interact causing additional dipoles. This means that a single Lorentz oscillator is no longer sufficient to describe the optical response of the film.

\subsubsection{DRUdE MODEL}

For unbound electrons there is no restoring force present, which means that $\omega_{0}=0$. The Drude oscillator then follows from Eq. (5.3.9) as [73, 75, p. 53]

$$
\epsilon(\omega)=\epsilon_{\infty}-\frac{\omega_{p}^{2}}{\omega^{2}+i \Gamma \omega}
$$

where $\omega_{p}$ is the plasma frequency given as

$$
\omega_{p}=\sqrt{\frac{e^{2} N_{e}}{\epsilon_{0} m_{e}}} .
$$




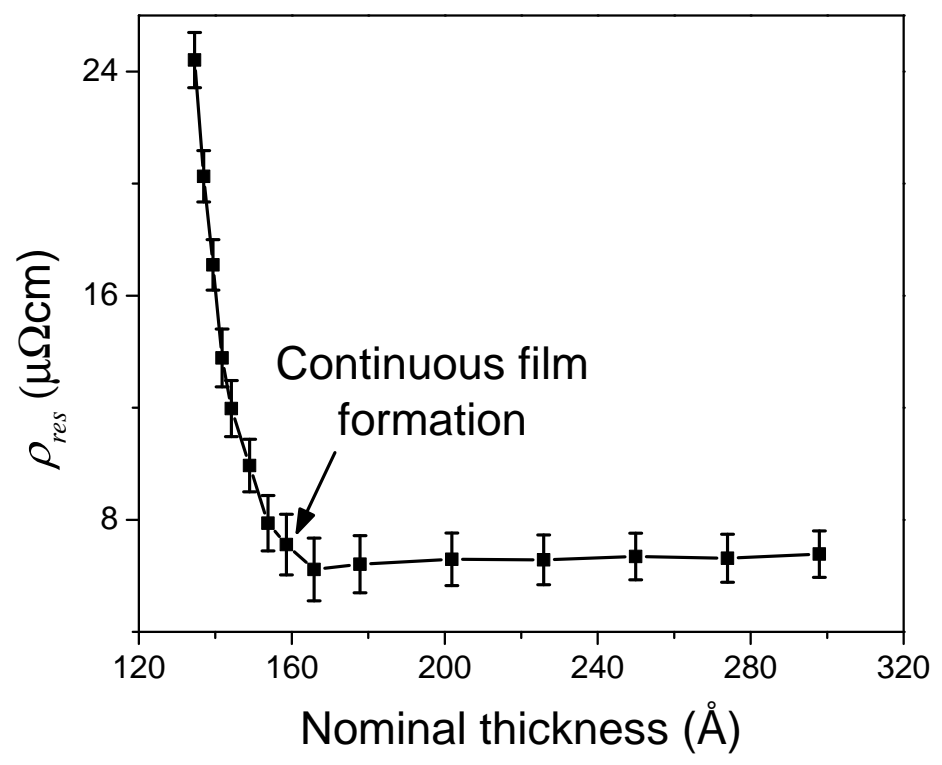

Fig. 5.3. Resistivity, $\rho_{\text {res }}$, as a function of nominal thickness (amount of deposited material). The point used to determine the continuous film formation is depicted in the figure.

By modelling the optical response of the film using the Drude model it is also possible to calculate the film resistivity $\rho_{\text {res }}$ as [73]

$$
\rho_{\text {res }}=\frac{\Gamma}{\epsilon_{0} \omega_{p}^{2}}
$$

This is done for continuous and close to continuous films in Paper 1 and 2 in order to monitor the evolution of $\rho_{\text {res }}$. From the latter the transition to a continuous film is extracted as the point where a steady-state value is reached [77], as indicated in Fig. 5.3.

\subsubsection{DOREMUS METHOD}

In 1966 Doremus [78] developed a method that determines the area fraction, $Q$, of a non-continuous metal film that covers a substrate depending on the maximum absorption of the film. In the derivation, the film was described as 
a collection of separated islands embedded in a dielectric medium using the Maxwell-Garnett [79] effective medium approximation. By considering an analytical expression of the film absorption Doremus found that a maximum occurred when [78]

$$
\epsilon_{1}=-\frac{(2+Q) n_{2}^{2}}{1-Q},
$$

where $\epsilon_{1}$ is the real part of the bulk complex dielectric function of the island material taken at the maximum absorption energy and $n_{2}$ the refractive index of the substrate. This simple relation has been shown to yield excellent agreement with experiments for $Q$ values in the range $19-63 \%$ [80].

In Paper 2, Eq. (5.3.13) is utilized to monitor the evolution of $Q$ for Ag films consisting of separated islands. The optical response of the films are modelled by a single Lorentz oscillator (see Sec. 5.3.1.1) where the energy at which maximum absorption occurs is given by $\omega_{0}$. In this way, $\epsilon_{1}$ in Eq. (5.3.13) could be extracted from reference data in Ref. [81] as $\epsilon_{1}=\epsilon_{1}\left(\omega_{0}\right)$.

\subsubsection{ARWin-Aspnes Method}

Arwin-Aspnes method is a graphical method that concurrently determines film thickness, $d$, and $\tilde{\epsilon}$ [82]. The derivation assumes that $\rho=\rho(\tilde{\epsilon}, d)$, i.e., that all other relevant parameters ( $\tilde{\epsilon}$ of substrate and ambient as well as energy and incidence angle of the light) are known. An approximative solution can then be found by expanding $\rho(\tilde{\epsilon}, d)$ to the first order around a hypothetical thickness $\langle d\rangle$, which is close to $d$, according to

$$
\rho(\tilde{\epsilon}, d)=\rho(\langle\tilde{\epsilon}\rangle,\langle d\rangle)+\frac{\partial \rho}{\partial d}(d-\langle d\rangle)+\frac{\partial \rho}{\partial \tilde{\epsilon}}(\tilde{\epsilon}-\langle\tilde{\epsilon}\rangle)
$$

As is evident from Eq. (5.3.14), $\rho(\tilde{\epsilon}, d)=\rho(\langle\tilde{\epsilon}\rangle,\langle d\rangle)$ if [82]

$$
\langle\tilde{\epsilon}\rangle=\tilde{\epsilon}+(d-\langle d\rangle) \frac{\partial \rho / \partial d}{\partial \rho / \partial \tilde{\epsilon}} .
$$

This, in turn, means that if $\langle d\rangle=d$ (the guessed thickness is correct) it is also valid that $\langle\tilde{\epsilon}\rangle=\tilde{\epsilon}$. Consequently, both $d$ and $\tilde{\epsilon}$ are determined at the same time. 
A prerequisite for this method to work is that an optical feature from the substrate is present within the measured $\omega$ range. In that case, the feature shows up as an artifact in $\langle\tilde{\epsilon}\rangle$ if $\langle d\rangle$ is not the correct thickness, due to that the last term in Eq. (5.3.15) is nonzero. An example of $\left\langle\epsilon_{1}\right\rangle$ and $\left\langle\epsilon_{2}\right\rangle$ for different $\langle d\rangle$ values of a Ag film is given in Fig. 5.4. As can be seen, a thickness of $140 \AA$ minimizes the influence of the substrate feature around $1.9 \mathrm{eV}$. Further refinement yields a thickness of $143 \AA$.

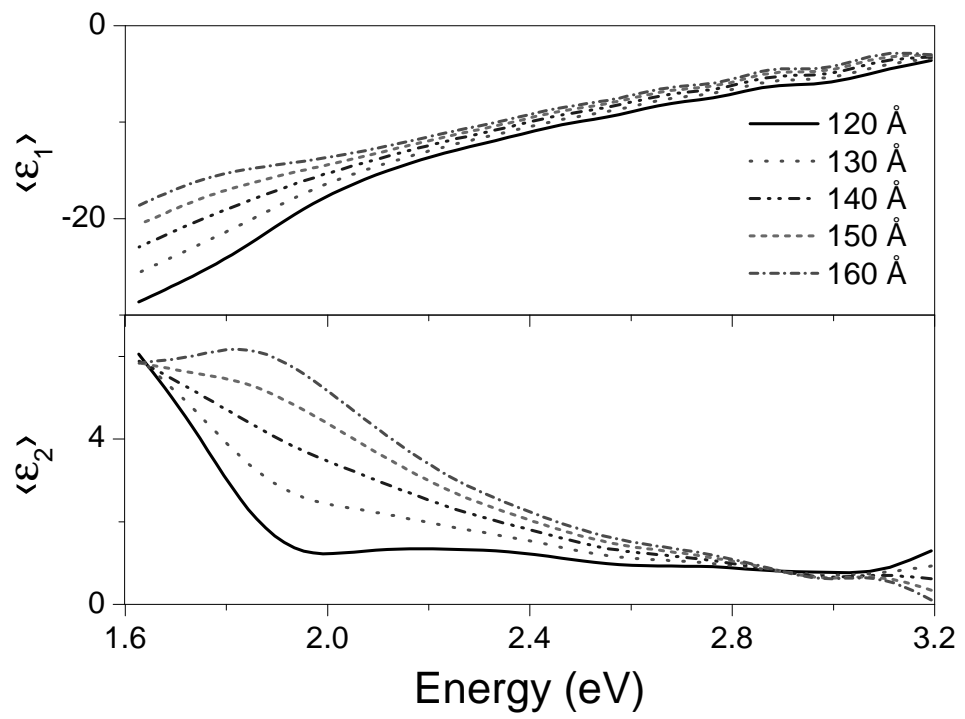

Fig. 5.4. Thickness determination using Arwin-Aspnes method. As can be seen, a thickness of $140 \AA$ minimizes the substrate feature around $1.9 \mathrm{eV}$.

By utilizing Arwin-Aspnes method for in situ real time measurements of film growth it is possible to determine the evolution of $\tilde{\epsilon}$, as displayed in Fig. 5.5 for a Ag film. The evolution of $\tilde{\epsilon}$ can, in turn, be employed to determine the time (or equivalently the nominal thickness) at which a film first starts to show metallic behaviour. This is accomplished by noting when $\epsilon_{1}$ becomes negative close to the near infrared region of the spectrum (low energy) [83], which corresponds to a film thickness between 100 and $109 \AA$ in Fig. 5.5. Refining the analysis results in a percolation thickness of $108 \AA$. This methodology is applied in Paper 2 to extract percolation thicknesses for growing Ag films and has previously been shown to be in good agreement with in situ resistivity measurements [84]. 


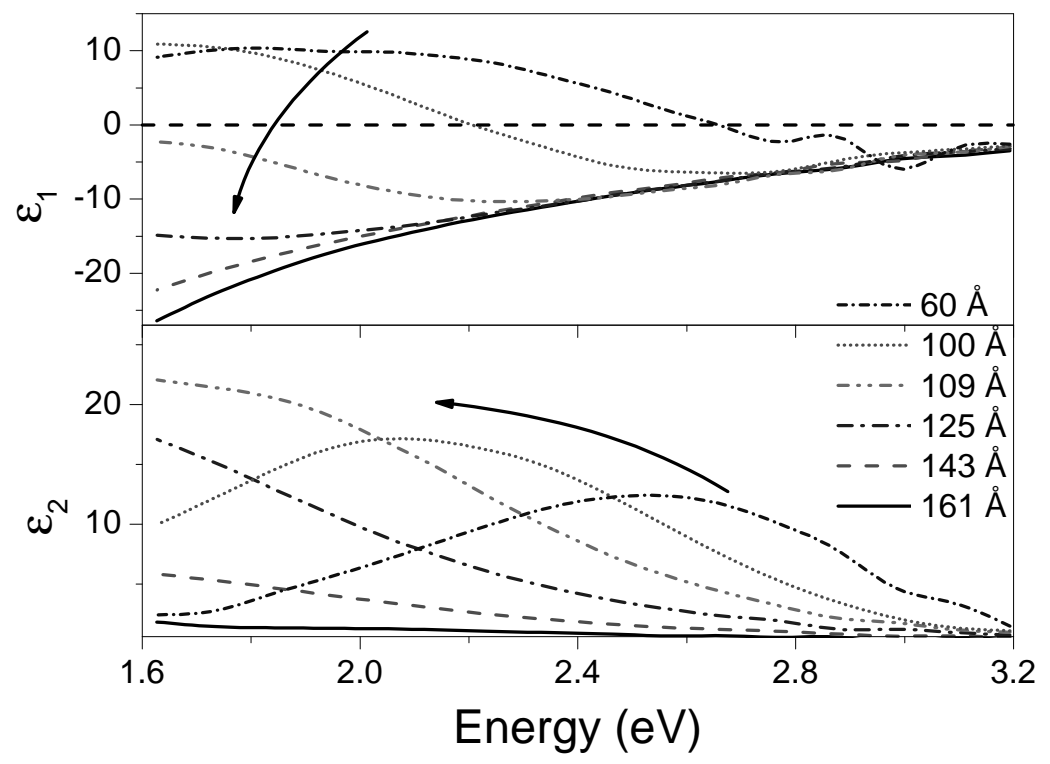

Fig. 5.5. Evolution of the complex dielectric function for a growing Ag film as extracted from Arwin-Aspnes method.

\subsection{Stress Measurements by Wafer Curvature}

Stress measurements by wafer curvature is a method that makes it possible to obtain film stresses in real time. The basic principle of the technique is that a stressed film, constrained on a substrate, exerts a force on the substrate causing it to bend until force equilibrium is reached. The radius of curvature, $R$, of the bended substrate can then be related to the film stress, $\sigma$, through the Stoney equation or a modified Stoney equation depending on type of substrate used. In the case of $\mathrm{Si}(100)$, as used in this thesis work, the modified Stoney equation is given by [85]

$$
\sigma d=\frac{M h^{2}}{6 R}
$$

where $d$ is the film thickness, $M$ the biaxial modulus of the substrate and $h$ the thickness of the substrate. As seen in Eq. (5.4.1) no specific film properties are required, except for the film thickness, or similarly, in the case of real time measurements, the deposition rate. Measurements of $R$ can be done in various 


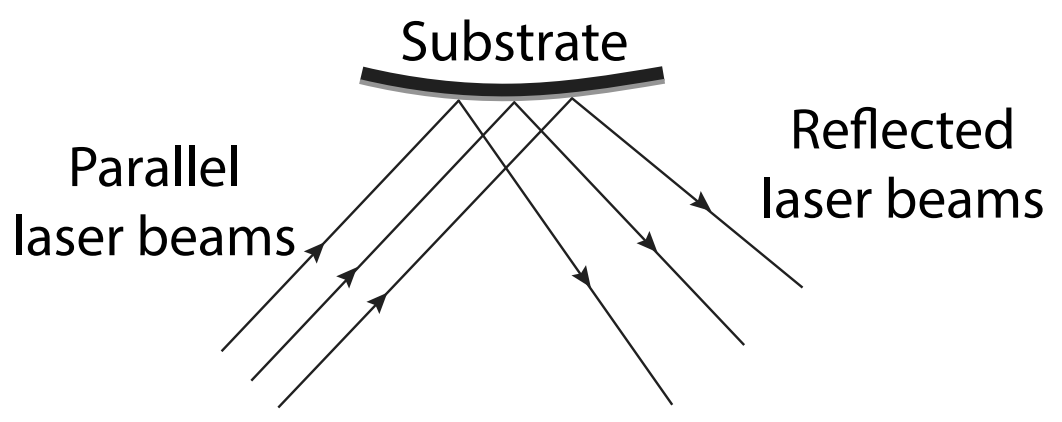

Fig. 5.6. Principles of stress measurements by wafer curvature. The incoming laser beams are reflected at different angles due to the curved substrate.

ways, where the one employed in Paper 1 to determine the formation of continuous Ag films uses parallel laser beams that are reflected from the substrate surface onto a CCD camera, as illustrated in Fig. 5.6. The curved substrate causes the reflected beams to deflect, which makes it possible to determine $R$ by comparing the spacing between the outgoing beams with the spacing of the reflected beams at the CCD camera.

\subsection{Scanning Electron Microscopy}

Scanning electron microscopy (SEM) is a widely used imaging technique for thin films owing to its high resolution capabilities ( $\sim$ nanometer scale is possible [86, p. 2]) together with easy handling and data interpretation. With the use of SEM it is possible to gain information such as microstructure, topography and chemical composition, from the film.

In order to obtain a micrograph, an electron beam is accelerated (typically by a few to tens of $\mathrm{kV}$ ) and focused to a small spot on the surface of the specimen. As the electrons impinge on the surface some of them are backscattered, while others can give rise to secondary electrons. By raster scanning the electron beam across an area of interest and at the same time detecting the intensity of either backscattered or secondary electrons an image is formed in a pixel-bypixel fashion. 
Backscattered electrons are caused by elastic collisions between incoming electrons and atoms in the sample, meaning that an incoming electron's trajectory changes due to attraction from the nuclei. As a consequence, electrons backscatter more for heavier atoms, which, thus, generates elemental contrast in the micrograph. On the contrary, secondary electrons are due to inelastic scattering and since their energies are comparatively low they originate from the near surface region. This means that they carry more information about the surface structure.

In Paper 3, SEM is used as characterization technique to measure tilt angles of the columnar microstructure in off-normally grown films as well as the corresponding film thickness by investigating cross-sectional samples.

\subsection{Atomic Force Microscopy}

Atomic force microscopy (AFM) is a type of scanning probe microscopy used in various types of fields to study surfaces. Today, it is routinely used to gain topographical information of surfaces.

The AFM employs a cantilever with a very sharp tip that is brought close to a specimen's surface, where the force acting on the cantilever is measured using a laser (see Fig. 5.7). If the tip is positioned at atomic distances from the surface, the cantilever experiences a repulsive force and the AFM is said to operate in contact mode. By raster scanning the surface at a constant cantilever force, achieved by adjusting the lateral tip position, a topographical map of the surface is acquired. A downside with contact mode is that the surface might be damaged as the tip is dragged across. Therefore, a non contact mode was developed, where the cantilever instead oscillates at or near its resonant frequency above the surface. This causes the tip to experience attractive van der Waals forces from the surface that decreases the oscillations. A topographical image can then be created in the same manner as in contact mode, but by instead keeping the oscillation frequency or amplitude constant. This makes it possible 


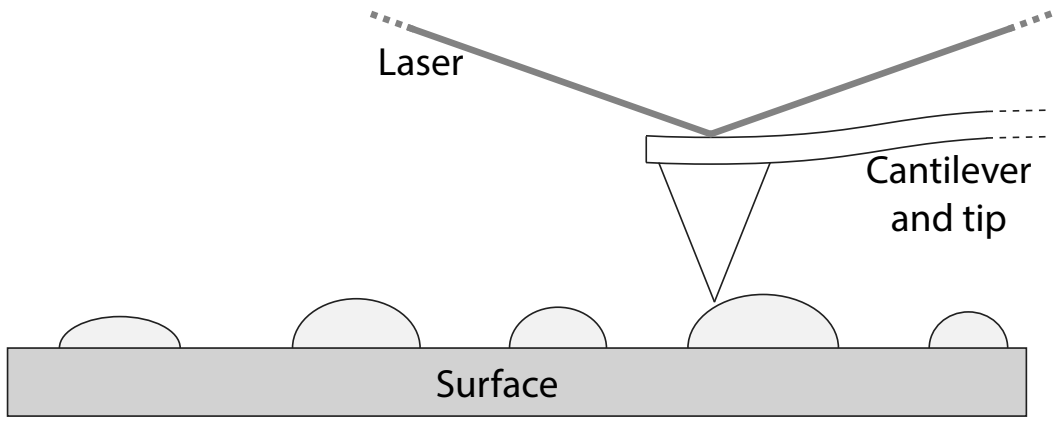

Fig. 5.7. Basic principles of atomic force microscopy (AFM).

to image soft surfaces (e.g., organic specimens) without any induced damage. However, if used at ambient conditions where, e.g., water easily condenses on the surface non-contact mode AFM yields the topography of the contaminant instead of the surface. These problems are circumvented by using a combination of the two operation modes where the tip is alternately brought into contact with the surface and then lifted up from the surface to avoid damage creation. This is referred to as tapping mode and is the operation mode utilized in Paper 2 to study the morphology of thin Ag films.

\subsection{X-ray Reflectometry}

X-ray reflectometry (XRR) is a non-destructive analysis technique that is widely used to determine properties, such as thickness, density and roughness, of thin films.

The specimen under investigation is irradiated with X-rays at gracing incidence. As long as the incidence angle is small total reflection occurs at the surface, but above a critical angle some of the X-rays penetrate into the specimen. Inside the sample additional interfaces (e.g., between film and substrate) are encountered, giving rise to both reflection and transmission of the incident beam. By detecting the specular reflected X-rays at different incident angles an interference pattern is obtained (see Fig. 5.8). The latter is then modelled in 
order to determine the desired properties. This technique is used in Paper 1 to determine deposition rates needed for the stress measurements (see Sec. 5.4).

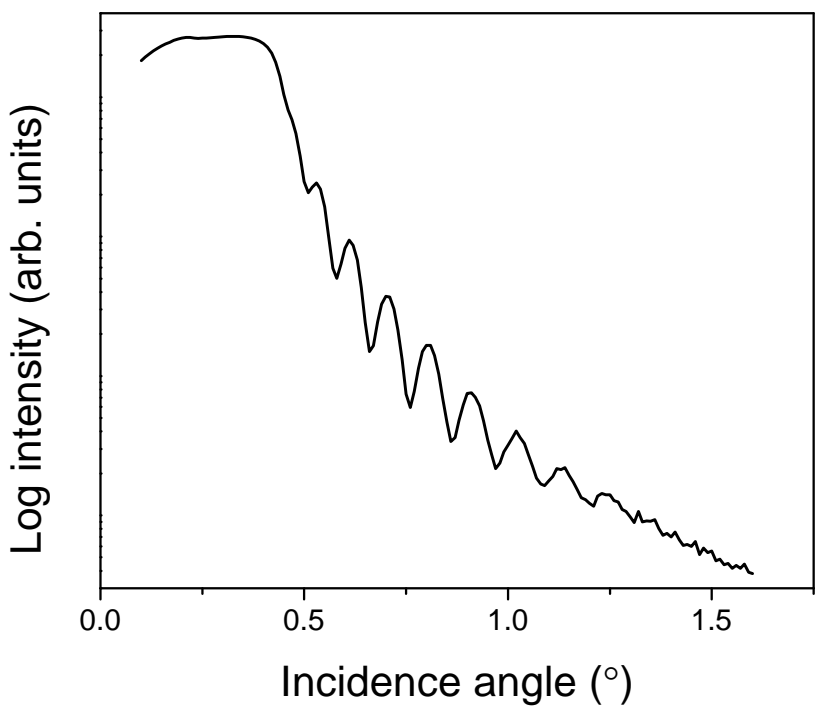

Fig. 5.8. Typical X-ray reflectivity (XRR) pattern. 


\section{CHAPTER 6}

\section{SumMARY OF APPENDEd PAPERS}

In Paper 1 we show that it is possible to generate pulsed vapor fluxes with a pulsing frequency that corresponds to that applied to the cathode in the HiPIMS process. In situ film growth monitoring is then used to determine the effect of the pulsed deposition fluxes on the formation of a continuous Ag film when grown on $\mathrm{SiO}_{2}$. The nominal thickness at which the latter occurs is shown to decrease when increasing the instantaneous deposition rate and the energy of the deposition flux as well as when only increasing the pulsing frequency (i.e., maintaining constant energetics of the flux as well as the same instantaneous rate within each pulse). The latter means that the effect of the vapor flux time domain is separated from contributions arising from energetics and instantaneous rates. By estimating adatom lifetime and island coalescence time it is suggested that both an increased island density and a retarded island coalescence may be responsible for the observed decrease of the continuous film formation. The influence of the pulsing frequency on the growth evolution of Ag films all the way from nucleation to the formation of a continuous film is further investigated in Paper 2. It is shown that a pulsing frequency dependence exists for characteristic growth transitions (elongation transition, percolation and continuous film formation), where the nominal transition thickness decreases with increasing pulsing frequency up to a certain frequency after which the thickness stays constant. This behaviour is demonstrated to be solely determined by the 
interplay between the pulsed deposition flux, as set by the pulsing frequency, and the time required for island coalescence to be completed.

The influence of highly ionized fluxes on the off-normal growth evolution is systematically investigated in Paper 3. The study is performed using two different metals, $\mathrm{Cu}$ and $\mathrm{Cr}$, that exhibit different diffusivities. For the high diffusivity case $(\mathrm{Cu})$, the tilted columnar microstructure is determined to be positioned closer to the substrate surface normal as the ionization degree of the deposition flux increases. This occurs since a larger fraction of the deposited species are deflected towards the substrate normal due to the potential difference between plasma and substrate. At the same time the ion energies increase, which can cause an enhanced directional diffusion towards the shadowed regions on the backside of the column tips and further raise the columnar structure. A large difference in column tilt is also observed between $\mathrm{Cu}$ and $\mathrm{Cr}$, where the Cr columns are positioned closer to the substrate surface normal. This is suggested to be due to different shadowing conditions caused by different island densities. Moreover, the column tilt of the $\mathrm{Cr}$ films is only found to be influenced by the ionized fluxes when certain nucleation characteristics are present. 


\section{CHAPTER 7}

\section{Future Research Possibilities}

The work presented in Papers 1 and 2 concerns growth evolution of Ag on $\mathrm{SiO}_{2}$, which is well-known to grow in a three-dimensional fashion (VolmerWeber type growth). The scaling behaviour presented in Paper 2 could, however, change for the case of two-dimensional growth. One possibility to extend the present work could thus be to use an epitaxial system and investigate its scaling behaviour. Another possibility could be to use a metal with a lower adatom diffusivity that might enable different nucleation regimes when pulsing the deposition flux (as described in Sec. 2.1.2). This may also affect the scaling behaviour. Moreover, Ag nanoparticles are commonly used owing to their optical properties that arise when exciting surface plasmons. By altering size and density of the particles when changing the vapor flux characteristics it could be possible to tune the corresponding optical properties.

In Paper 3 the microstructural evolution during off-normal film growth is investigated. When performing depositions at an off-normal geometry the crystallographic directions of the grains in the film can align up with each other to form preferred out-of-plane and in-plane orientations. This is known as biaxial texture. How and to what extent the biaxial texture is influenced by the highly ionized fluxes as well as understanding the underlying physical mechanisms would be an interesting continuation of the present work. 


\section{REFERENCES}

[1] E. D. Nicholson. Gold Bull. 12, 161-166 (1979).

[2] C. Schaefer, G. Bräuer, and J. Szczyrbowski. Surf. Coat. Technol. 93, 37-45 (1997).

[3] M. Ohring. Materials science of thin films. Academic Press, San Diego, 2nd edition (2002).

[4] I. Petrov, P. B. Barna, L. Hultman, and J. E. Greene. J. Vac. Sci. Technol. A 21, S117-S128 (2003).

[5] J. E. Greene, S. A. Barnett, J.-E. Sundgren, and A. Rockett. Low-energy ion/surface interactions during film growth from the vapor phase. In T. Itoh (editor), Ion beam assisted film growth, chapter 5, pages 101-152. Elsevier Inc., Amsterdam (1989).

[6] D. Dobrev. Thin Solid Films 92, 41-53 (1982).

[7] E. Kay. J. Vac. Sci. Technol. A 6, 3074-3081 (1988).

[8] M. Holber, S. Logan, H. J. Grabarz, J. T. C. Yeh, J. B. O. Caughman, A. Sugerman, and F. E. Turene. J. Vac. Sci. Technol. A 11, 2903-2910 (1993).

[9] S. M. Rossnagel. J. Vac. Sci. Technol. B 16, 2585-2608 (1998).

[10] E. Klawuhn, G. C. D'Couto, K. A. Ashtiani, P. Rymer, M. A. Biberger, and K. B. Levy. J. Vac. Sci. Technol. A 18, 1546-1549 (2000).

[11] P. Jensen and B. Niemeyer. Surf. Sci. 384, L823-L827 (1997). 
[12] Q. Y. Zhang and P. K. Chu. Surf. Coat. Technol. 158-159, 247-252 (2002).

[13] M. Schmid, C. Lenauer, a. Buchsbaum, F. Wimmer, G. Rauchbauer, P. Scheiber, G. Betz, and P. Varga. Phys. Rev. Lett. 103, 076101 (2009).

[14] J. Warrender and M. Aziz. Phys. Rev. B 76, 045414 (2007).

[15] V. Kouznetsov, K. Macák, J. M. Schneider, U. Helmersson, and I. Petrov. Surf. Coat. Technol. 122, 290-293 (1999).

[16] K. Sarakinos, J. Alami, and S. Konstantinidis. Surf. Coat. Technol. 204, 16611684 (2010).

[17] T. Michely and J. Krug. Islands, mounds and atoms: Patterns and processes in crystal growth far from equilibrium. Springer, Berlin (2004).

[18] R. Ditchfield and E. Seebauer. Phys. Rev. Lett. 82, 1185-1188 (1999).

[19] D. Adamovic, E. P. Münger, V. Chirita, L. Hultman, and J. E. Greene. Appl. Phys. Lett. 86, 211915 (2005).

[20] X. W. Zhou and H.N.G. Wadley. Surf. Sci. 431, 42-57 (1999).

[21] P. M. Martin. Handbook of deposition technologies for films and coatings. Elsevier Inc., Oxford, 3rd edition (2010).

[22] Z. Zhang and M. G. Lagally. Science 276, 377-383 (1997).

[23] D. W. Pashley. J. Vac. Sci. Technol. 3, 156-166 (1966).

[24] F. A. Nichols and W. W. Mullins. J. Appl. Phys. 36, 1826-1835 (1965).

[25] G. Jeffers, M. A. Dubson, and P. M. Duxbury. J. Appl. Phys. 75, 5016-5020 (1994).

[26] J. Wen, S. Chang, J. Burnett, J. Evans, and P. Thiel. Phys. Rev. Lett. 73, 2591-2594 (1994). 
[27] R. Hull and J. C. Bean. Principles and concepts of strained-layer epitaxy. In T. P. Pearsall (editor), Strained-layer superlattices: Materials science and technology, chapter 1, pages 1-72. Academic Press Inc., San Diego (1991).

[28] L. Vitos, A. V. Ruban, H. L. Skriver, and J. Kollar. Surf. Sci. 411, 186-202 (1998).

[29] H. Brune, K. Bromann, H. Röder, Kl. Kern, J. Jacobsen, P. Stoltze, K. Jacobsen, and J. Norskov. Phys. Rev. B 52, R14380-R14383 (1995).

[30] K. Morgenstern, G. Rosenfeld, E. Lægsgaard, F. Besenbacher, and G. Comsa. Phys. Rev. Lett. 80, 556-559 (1998).

[31] M. A. Garcia. J. Phys. D: Appl. Phys. 44, 283001 (2011).

[32] D. R. Rolison. Science 299, 1698-1701 (2003).

[33] Y. Shiratsuchi, M. Yamamoto, and S. Bader. Prog. Surf. Sci. 82, 121-160 (2007).

[34] Y. Zhao, Y. He, and C. Brown. Appl. Phys. Lett. 100, 033106 (2012).

[35] K. Robbie, J. C. Sit, and M. J. Brett. J. Vac. Sci. Technol. B 16, 1115-1122 (1998).

[36] M. Saraiva and D. Depla. J. Appl. Phys. 111, 104903 (2012).

[37] J. M. García-Martín, R. Alvarez, P. Romero-Gomez, A. Cebollada, and A. Palmero. Appl. Phys. Lett. 97, 173103 (2010).

[38] N. G. Nakhodkin and A. I. Shaldervan. Thin Solid Films 10, 109-122 (1972).

[39] T. Hashimoto, K. Okamoto, K. Hara, M. Kamiya, and H. Fujiwara. Thin Solid Films 91, 145-154 (1982).

[40] K. Okamoto, T. Hashimoto, K. Hara, M. Kamiya, and H. Fujiwara. Thin Solid Films 147, 299-311 (1987).

[41] K. Okamoto and K. Itoh. Jpn. J. Appl. Phys. 44, 1382-1388 (2005). 
[42] M. M. Hawkeye and M. J. Brett. J. Vac. Sci. Technol. A 25, 1317-1335 (2007).

[43] J. Alami, P. O. A. Persson, D. Music, J. T. Gudmundsson, J. Bohlmark, and U. Helmersson. J. Vac. Sci. Technol. A 23, 278-280 (2005).

[44] J. A. Floro, E. Chason, R. C. Cammarata, and D. J. Srolovitz. MRS Bull. 27, 19-25 (2002).

[45] D. Flötotto, Z. M. Wang, L. P. H. Jeurgens, E. Bischoff, and E. J. Mittemeijer. J. Appl. Phys. 112, 043503 (2012).

[46] E. Chason. Thin Solid Films 526, 1-14 (2012).

[47] E. Chason, J. W. Shin, S. J. Hearne, and L. B. Freund. J. Appl. Phys. 111, 083520 (2012).

[48] D. Magnfält, G. Abadias, and K. Sarakinos. Appl. Phys. Lett. 103, 051910 (2013).

[49] F. F. Chen. Introduction to plasma physics and controlled fusion. Springer, New York, 2nd edition (1984).

[50] J. T. Gudmundsson, J. Alami, and U. Helmersson. Appl. Phys. Lett. 78, 3427 (2001).

[51] J. T. Gudmundsson, J. Alami, and U. Helmersson. Surf. Coat. Technol. 161, 249-256 (2002).

[52] A. Hecimovic, K. Burcalova, and A. P. Ehiasarian. J. Phys. D: Appl. Phys. 41, 095203 (2008).

[53] A. D. Pajdarová, J. Vlček, P. Kudláček, and J. Lukáš. Plasma Sources Sci. Technol. 18, 025008 (2009).

[54] V. Stranak, S. Drache, M. Cada, Z. Hubicka, M. Tichy, and R. Hippler. Contrib. Plasma Phys. 51, 237-245 (2011).

[55] I. Petrov, F. Adibi, J. E. Greene, L. Hultman, and J.-E. Sundgren. Appl. Phys. Lett. 63, 36-38 (1993). 
[56] I. Petrov, F. Adibi, J. E. Greene, W. D. Sproul, and W. D. Munz. J. Vac. Sci. Technol. A 10, 3283-3287 (1992).

[57] C. Christou and Z. H. Barber. J. Vac. Sci. Technol. A 18, 2897-2907 (2000).

[58] U. Helmersson, M. Lattemann, J. Bohlmark, A. P. Ehiasarian, and J. T. Gudmundsson. Thin Solid Films 513, 1-24 (2006).

[59] J. Alami, S. Bolz, and K. Sarakinos. J. Alloys Compd. 483, 530-534 (2009).

[60] A. Anders. Surf. Coat. Technol. 204, 2864-2868 (2010).

[61] D. Lundin and K. Sarakinos. J. Mater. Res. 27, 780-792 (2012).

[62] J. T. Gudmundsson, N. Brenning, D. Lundin, and U. Helmersson. J. Vac. Sci. Technol. A 30, 030801 (2012).

[63] J. Bohlmark, J. T. Gudmundsson, J. Alami, M. Latteman, and U. Helmersson. IEEE Trans. Plasma Sci. 33, 346-347 (2005).

[64] J. Bohlmark, J. Alami, C. Christou, A. P. Ehiasarian, and U. Helmersson. J. Vac. Sci. Technol. A 23, 18-22 (2005).

[65] N. Brenning, D. Lundin, T. Minea, C. Costin, and C. Vitelaru. J. Phys. D: Appl. Phys. 46, 084005 (2013).

[66] A. Anders, M. Panjan, R. Franz, J. Andersson, and P. Ni. Appl. Phys. Lett. 103, 144103 (2013).

[67] F. Mitschker, M. Prenzel, J. Benedikt, and A. von Keudell. J. Phys. D: Appl. Phys. 45, 402001 (2012).

[68] W. Breilmann, C. Maszl, J. Benedikt, and A. von Keudell. J. Phys. D: Appl. Phys. 46, 485204 (2013).

[69] A. F. Voter. Introduction to the kinetic Monte Carlo method. In K. E. Sickafus, E. A. Kotomin, and B. P. Uberuaga (editors), Radiation effects in solids, chapter 1, pages 1-23. IOS Press/Springer, Amsterdam (2007). 
[70] J. Carrey and J.-L. Maurice. Phys. Rev. B 63, 245408 (2001).

[71] G. Sauerbrey. Z. Phys. 155, 206-222 (1959).

[72] C. Kittel. Introduction to solid state physics. John Wiley \& Sons Inc., New York (2005).

[73] T. W. H. Oates, H. Wormeester, and H. Arwin. Prog. Surf. Sci. 86, 328-376 (2011).

[74] S. Logothetidis. In situ and real-time spectroscopic ellipsometry studies: Carbon based and metallic $\mathrm{TiN}_{x}$ thin films growth. In H. S. Nalwa (editor), Handbook of thin films, vol. 2: Characterization and spectroscopy of thin films, chapter 6, pages 277-330. Academic Press, San Diego (2002).

[75] F. Wooten. Optical properties of solids. Academic Press, New York (1972).

[76] H. Ehrenreich and H. R. Philipp. Phys. Rev. 128, 1622-1629 (1962).

[77] P. Patsalas and S. Logothetidis. J. Appl. Phys. 93, 989-998 (2003).

[78] R. H. Doremus. J. Appl. Phys. 37, 2775-2781 (1966).

[79] D. E. Aspnes. Thin Solid Films 519, 2571-2574 (2011).

[80] R. Doremus. Thin Solid Films 326, 205-210 (1998).

[81] P. B. Johnson and R. W. Christy. Phys. Rev. B 6, 4370-4379 (1972).

[82] H. Arwin and D. E. Aspnes. Thin Solid Films 113, 101-113 (1984).

[83] H. Nguyen, I. An, and R. W. Collins. Phys. Rev. B 47, 3947-3965 (1993).

[84] T. W. H. Oates, D. R. McKenzie, and M. M. M. Bilek. Phys. Rev. B 70, 195406 (2004).

[85] G. C. A. M. Janssen, M. M. Abdalla, F. van Keulen, B. R. Pujada, and B. van Venrooy. Thin Solid Films 517, 1858-1867 (2009). 
[86] J. Goldstein, D. Newbury, D. Joy, C. Lyman, P. Echlin, E. Lifshin, L Sawyer, and J Michael. Scanning electron microscopy and X-ray microanalysis. Kluwer Academic/Plenum, New York, 3rd edition (2003). 



\section{Papers}

The articles associated with this thesis have been removed for copyright reasons. For more details about these see:

http://urn.kb.se/resolve?urn=urn:nbn:se:liu:diva-103921 